\title{
Horizontal simulation grids as alternative to structure-based grids for thin oil-zone problems: a comparison study on a Troll segment
}

\author{
Øystein Pettersen
}

Received: 24 January 2011 / Accepted: 14 June 2011 / Published online: 7 July 2011

(C) The Author(s) 2011. This article is published with open access at Springerlink.com

\begin{abstract}
The layering in reservoir simulation grids is often based on the geology, e.g., structure tops. In this paper we investigate the alternative of using horizontal layers, where the link to the geology model is by the representation of the petrophysics alone. The obvious drawback is the failure to honor the structure in the grid geometry. On the other hand, a horizontal grid will honor the initial fluid contacts perfectly, and horizontal wells can also be accurately represented. Both these issues are vital in thin oil-zone problems, where horizontal grids may hence be a viable alternative. To investigate this question, a number of equivalent simulation models were built for a segment of the Troll Field, both geology-based and horizontal, and various combinations of these. In the paper, it is demonstrated that the horizontal grid was able to capture the essentials of fluid flow with the same degree of accuracy as the geology-based grid, and near-well flow was considerably more accurate. For grids of comparable resolution, more reliable results were obtained by a horizontal grid than a geo-grid. A geo-grid with local grid refinement and a horizontal grid produced almost identical results, but the ratio of computing times was almost 20 in favor of the horizontal grid. In the one-phase regions of the reservoir, relatively coarse cells can be used without significant loss of accuracy.
\end{abstract}

Keywords Reservoir simulation - Thin oil-zone • Well modeling

$\varnothing$. Pettersen $(\bowtie)$

Centre for Integrated Petroleum Research, Bergen, Norway

e-mail: oystein.pettersen@uni.no

\section{Introduction}

The Troll Field is situated about $60 \mathrm{~km}$ west of the Norwegian West coast, at approx. $300 \mathrm{~m}$ water depth, and is operated by Statoil. It is primarily a gas field, but also contains a thin oil zone with thickness varying from zero to $26 \mathrm{~m}$. The use of horizontal wells has resulted in a successful recovery of this oil zone, which initially was regarded as challenging to produce due to coning and cusping of both water and gas-typical for thin oil zone production $[4,7,9,13,14]$.

Simulation of the Troll oil zone also represents a challenge, due to the combination thin oil zone and huge areal extent. A high-resolution grid is needed to capture fluid movement in the vicinity of the producing wells, while the size of the field prohibits such a grid field-wise. One successful compromise was presented by Henriquez, Apeland, Lie, and Cheshire [5], where they used local grid refinement (LGR) surrounding the wells and vertical equilibrium (VE) outside the LGR areas. Although good results were achieved there was (and still is) some uncertainty tied to the accuracy of the VE-to-LGR flow. In addition, the simulations were time-demanding, and were implemented on a parallel architecture [2].

The concept of corner-point gridding is widely used in the industry, and can perhaps be seen as an industry standard for grids where geological features as, e.g., structure horizons and faults are attempted adapted to the grid as faithfully as possible (such grids will be referred to as geo-grids in this paper). In a cornerpoint grid, all cells consist of eight corners, such that all corners with the same $(i, j)$-index lie on the same nonhorizontal straight line ("coordinate line"). The scheme allows for straightforward adaption of structure depths 
Table 1 Benefits and drawbacks of geo-grid vs. hor-grid: a priori intuitive feeling

\begin{tabular}{lll}
\hline & Geo-grid & Hor-grid \\
\hline Geological layering & Accurate & Only implicit \\
Petrophysics & Honors data & Approx. honors data \\
Fluid contacts & Approximate & Accurate \\
Contact movement & Approximate & Expected better \\
Well completions & Approximate & Can be accurate \\
General fluid flow & Simulator quality & Open question, topic of this paper \\
\hline
\end{tabular}

and fault throws from the geological model into the simulation grid, handling most not-too-complex structures, such that many (probably a majority) of the grids in present simulation models are closely adapted to the geological model.

A completely different approach would be to use a grid where all grid layers are horizontal, i.e., with identical corner depth for all cell tops in the same grid layer (denoted hor-grids in this paper). Such a grid would obviously be completely free-coupled from the structure geometry, which may be disadvantageous. On the other hand, the initial fluid contacts can be modeled exactly; hence a horizontal grid can be suitable when accurate modeling of fluid contact movement is an important factor. One important example is thinoil-zone problems, where the vertical distance from the gas-oil contact (GOC) or oil-water contact (OWC) to a producing well in the oil leg is small. For simulation of fluid flow in and near the oil leg, a high-resolution grid is required. For sloping structures, this enforces high resolution in most of the grid, as all or most of the layers pass through the oil leg somewhere; or alternatively, local grid refinement would have to be extensively used. Using a horizontal grid in a thin oil zone setting, the high-resolution part of the grid can be confined to the dynamic three-phase domain, roughly from somewhat above the initial GOC to somewhat below the OWC, which is a relatively small volume compared to the entire reservoir. In the gas and water zones, away from the contacts, the flow is essentially one-phase and high resolution is not critical.

A priori, the intuitive benefits and drawbacks of the two alternative grid formulations can be summarized as in Table 1.

The main disadvantage of a horizontal grid is the lack of alignment to the structural model, which historically has been seen as highly desired (whether this is a real or conceived drawback is attempted resolved in this paper). On the other hand, the approximate fluid contact description that is the consequence of sloping grid cells in a geo-grid can be a source of inaccuracy; especially if distances from the contact to well completions are small. An example of initial contact representation on the (sloping) grid is shown in Fig. 1 (taken from the BASEGEO model described below). The large number of grid layers passing through the oil leg can also be seen.

Provided the quality of the simulated fluid flow is acceptable on a horizontal grid, this kind of grid also has some computational advantages;

- The oil zone can be modeled with sufficiently thin layers, which can be confined to the oil zone alone, and hence does not increase the total number of cells significantly (high resolution only where needed).

- As all cell top/bottoms are horizontal, cell shapes will be regular, hence better suited for numerical computations.

- All cell-to-cell connections are regular, even across faults (no non-neighbor connections (NNCs) in corner-point grid terminology).

In Statoil and Norsk Hydro horizontal grids were used to model near-well flow on Troll already in the beginning of the 1990s; as an example, the gas production in a well test was successfully matched with a hor-grid model when no such match was achieved using a standard geo-grid. Later, the hor-grid methodology was used to study thin oil-zone problems on, e.g., the Sleipner field in the North Sea, Asgard on Haltenbanken, and Snøhvit outside northern Norway, with a large span in both fluid properties and oil zone thickness. All these studies were only reported

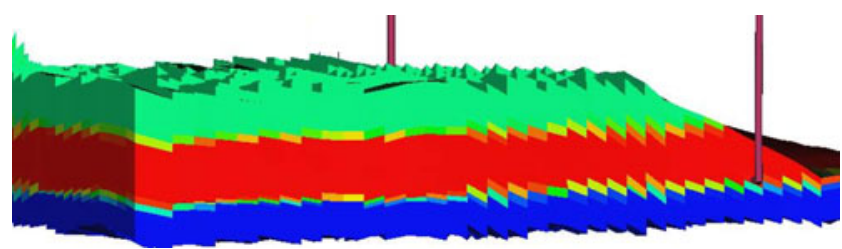

Fig. 1 Example of representation of fluid contacts on sloping grid cells. Green is gas, red oil, and blue water. Section taken from BASEGEO model. Only a part of the vertical section, including the oil leg, is shown 
in (confidential) internal reports. During the last few decades, such grids have been used in different contexts and with apparent success, e.g., Hsu [6] and Kabir et al. [7]. However, all these models were used as ad hoc tools, and no attempts were made to assess the quality of the horizontal grid models versus traditional geo-grids.

When Norsk Hydro AS (later Statoil) decided to build a new generation of Troll oil zone simulation models, the apparent success of the earlier horizontal grid models led to the decision to test and compare the performance of different kinds of gridding approaches on a representative segment of the field. The goal was to identify the strategy that would provide the best combination of reliable results and affordable computing time.

The Troll sands include an alternating pattern of dipping clean, high-permeability sands (C-sands) interbedded with micaceous and silty to fine-grained sands of significantly lower permeability (M-sands). Typical permeabilities are in the range $8-15 \mathrm{D}$ for the C-sands and 500-5,000 mD for the M-sands. Porosity is relatively high, with typical values ranging from 0.27 to 0.35 , and with values as high as 0.4 occurring in some areas. The deepest part of the reservoir is of poorer quality, with porosities $0.18-0.25$ and permeability down to $200 \mathrm{mD}$. Examples of the distribution and appearance of the C- and M-sands are shown in Figs. 2 and 5. Calcite cementation occurs in all lithologies over the entire field. The production scenario is to a large extent based on the principle of placing the horizontal wells in C-sands, slightly above the OWC, such that the $\mathrm{M}$-sands may act as partial barriers to gas inflow from above. An important aspect of the horizontal grid is
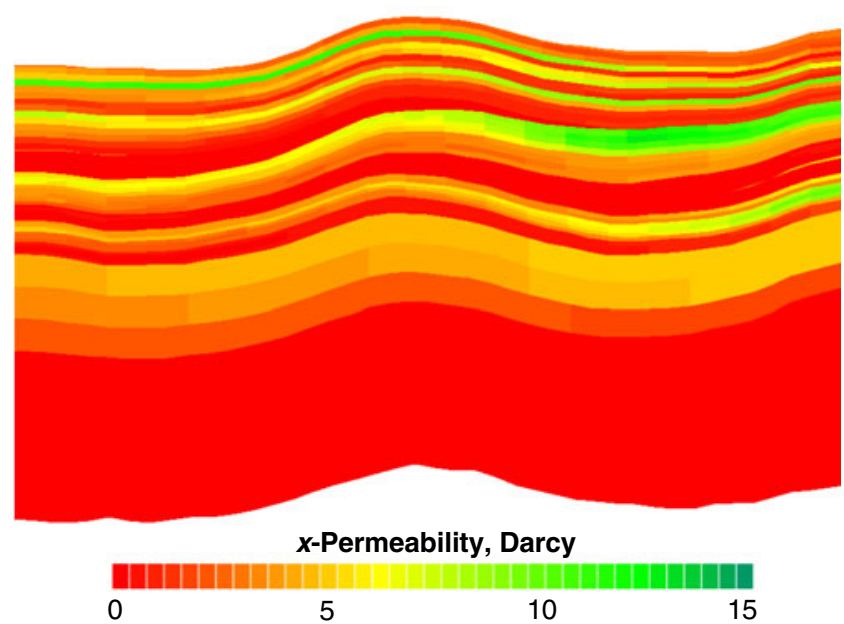

Fig. 2 Vertical cross-section of original geo-grid therefore the ability to reproduce the interbedding of $\mathrm{C}$ - and M-sands, and capture the gas flow pattern from the gas zone to the well perforations, as well as the fluid contacts movement through time.

\section{Geo-grids and hor-grids}

The horizontal grids in this paper were constructed by purpose-developed software, by direct sampling and rescaling of existing geo-grids. The basic geo-grid should be as closely adapted to the geologic model as possible. It is typically comprised of many more layers than would be used in a simulation model, to achieve high vertical resolution and hence improved rescaling of data to the horizontal grid (see below for details), i.e., the algorithm for constructing a hor-grid is to first build a traditional geo-grid with high vertical resolution, which is only used as basis for the hor-grid construction. Then, the horizontal grid is automatically constructed by the software according to user request, primarily the horizontal layer thicknesses and depths. The software constructs an almost complete input data deck for the ECLIPSE reservoir simulator [12], and once the basic geo-grid is in place each new hor-grid can be constructed in a few minutes. During the conversion from geo-grid to hor-grid, the original coordinate lines are left unchanged; hence, the areal definition of the horizontal grid cells is identical to the geo-grid.

It is also possible to construct a horizontal grid directly from the geologic model by existing commercial software, which was also done in this project. The purpose-developed program was however more efficient when many different realizations were needed,

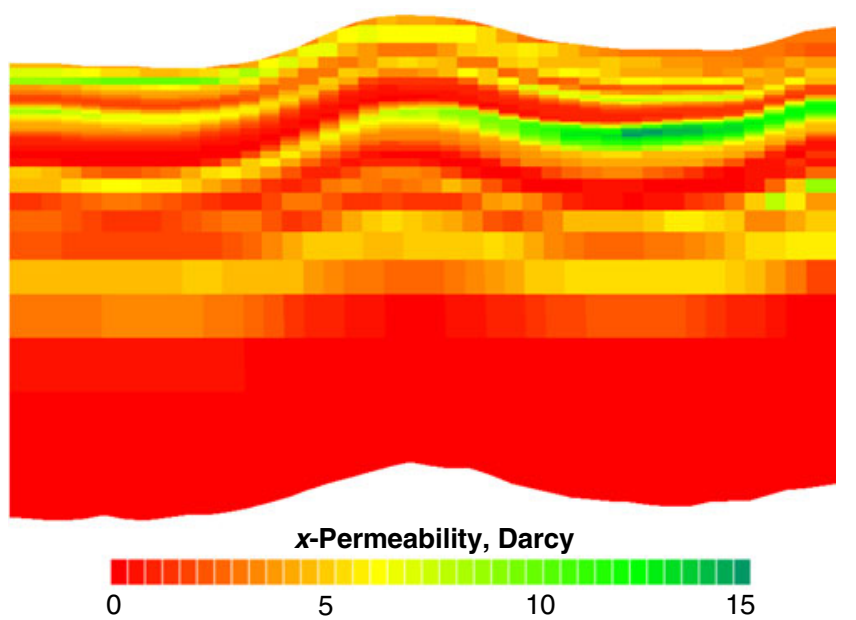

Fig. 3 Cross-section as in Fig. 2, true horizontal grid 


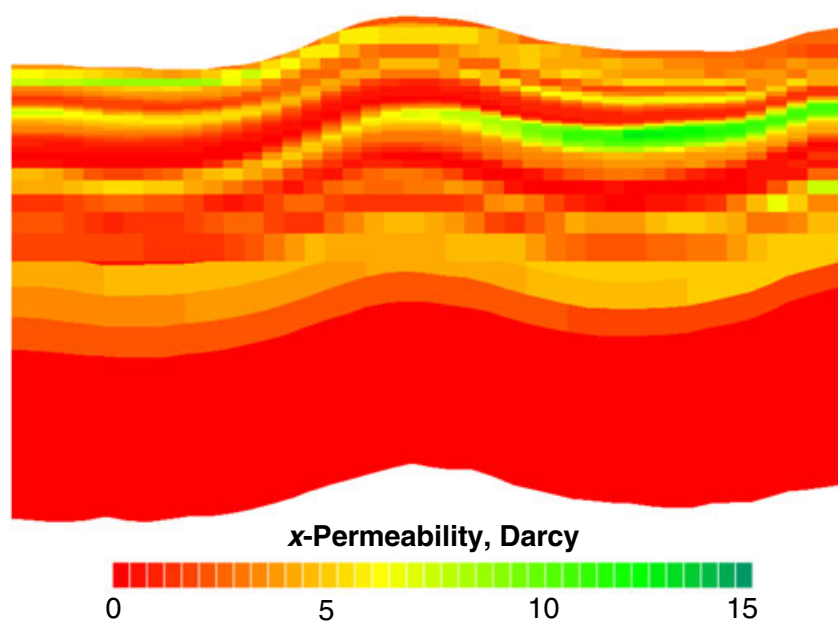

Fig. 4 Cross-section as in Fig. 2, hybrid grid

as in this project. In addition, the rescaling algorithm described below is only available in the purposedeveloped software.

The final grid can be either a pure horizontal grid, or a hybrid grid (combination of geo-based and horizontal layers). A general description of the target grid is,

1. $n_{\mathrm{T}}$ geo-based layers in the upper geo-grid zone.

2. $n_{\mathrm{H}}$ horizontal layers in the hor-layers zone.

3. $n_{\mathrm{B}}$ geo-based layers in the lower geo-grid zone.

A true horizontal grid is obtained when $n_{\mathrm{T}}=n_{\mathrm{B}}=0$, else a hybrid grid results.

Figures 2, 3, 4, 5 and 6 show examples of the different kinds of grid, including the permeability in the $x$ direction. All these figures were taken from the Troll segment model that is discussed in this paper.

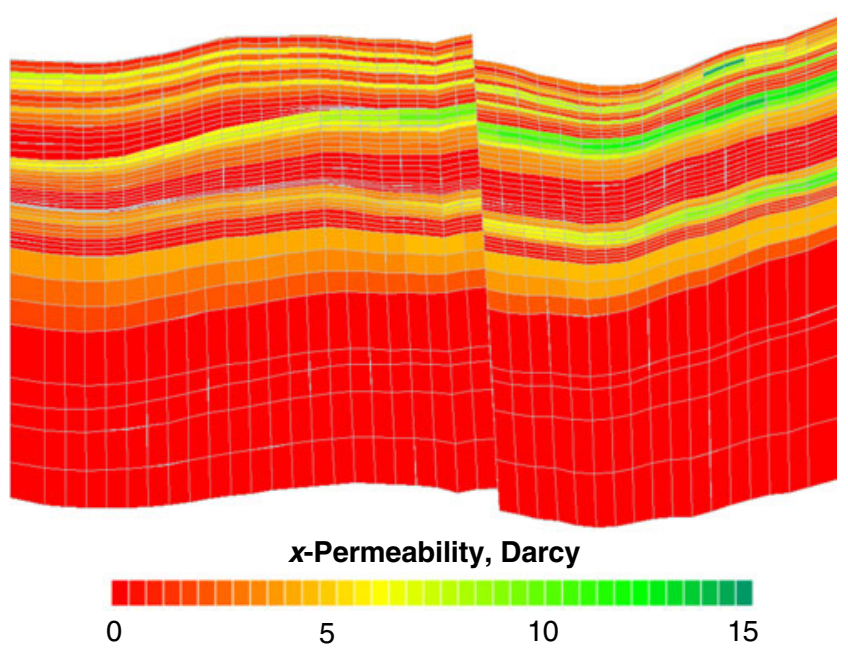

Fig. 5 Vertical cross-section including a fault, geo-grid

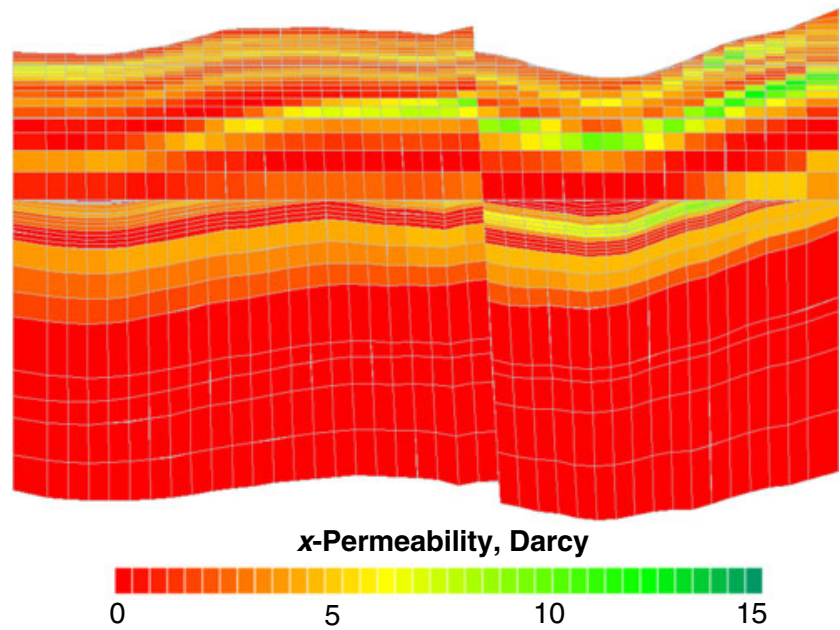

Fig. 6 Cross-section as in Fig. 5, hybrid grid

Note the handling of faults in the horizontal grid, visualized in Figs. 5 and 6. In a geo-grid, faults are geometrical discontinuities, which result in non-neighbor connections in a corner-point grid. In the horizontal grid, the fault is represented as a discontinuity in the petrophysics only-all geometric connections between grid cells across the fault are regular and continuous. Hence, no non-neighbor connections are present, and thereby no off-band elements in the coefficient matrix, which typically implies better convergence properties for the numerical solution.

Note also the representation of the high, respective low permeability zones. It is imperative that sand continuity is conserved in the horizontal grid for such zones.

\section{Rescaling geo-grids to hor-grids}

As the grid cell sizes in the geo-grid and the horizontal grid normally are of the same order of magnitude, mapping of parameters from the geo-grid to the horizontal grid is relatively straightforward, with a possible exception for the permeabilities. For completeness, we describe the rescaling procedure.

In any cell, its areal indices ( $i$ and $j$ ) will be the same on the geo-grid and the hor-grid. For the vertical index we use $K$ for the geo-grid layer, and $k$ for the hor-grid layer.

Looking at cell $(i, j, k)$ in the horizontal grid, in the general case $N$ geo-grid layers $\left(K=K_{1}, K_{2}, \ldots K_{N}\right)$ will pass through the cell, as in Fig. 7.

First the volumes of the part of the geo-layers that belong to the horizontal cell are evaluated, $V_{1}$, $V_{2}, \ldots, V_{N}$. Integer variables (typically fluid-in-place regions and other defined regions) are taken as the value 


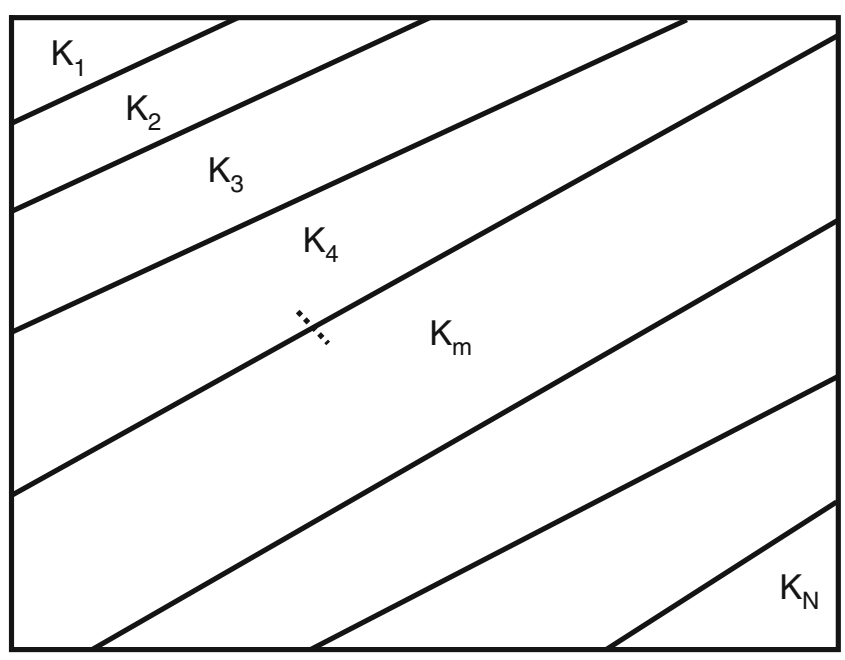

Fig. 7 Example of a horizontal grid cell, with $N$ geo-grid layers passing through the cell

belonging to the maximum of the $V_{m}, m=1, \ldots, N$. Properties requiring arithmetic average (porosity, netto-gross) are computed as standard volume-weighted averages.

\subsection{Rescaling of permeability}

Horizontal permeability $K_{h}$ is generally understood as the permeability in the direction along the layer, as the vector $K X^{\mathrm{Geo}}$ in Fig. 8. In a horizontal grid where the grid cells are not aligned to the geologic structures there is no tie to the structure direction, and the horizontal permeability will wrongly be interpreted as true horizontal. This is also in agreement with the

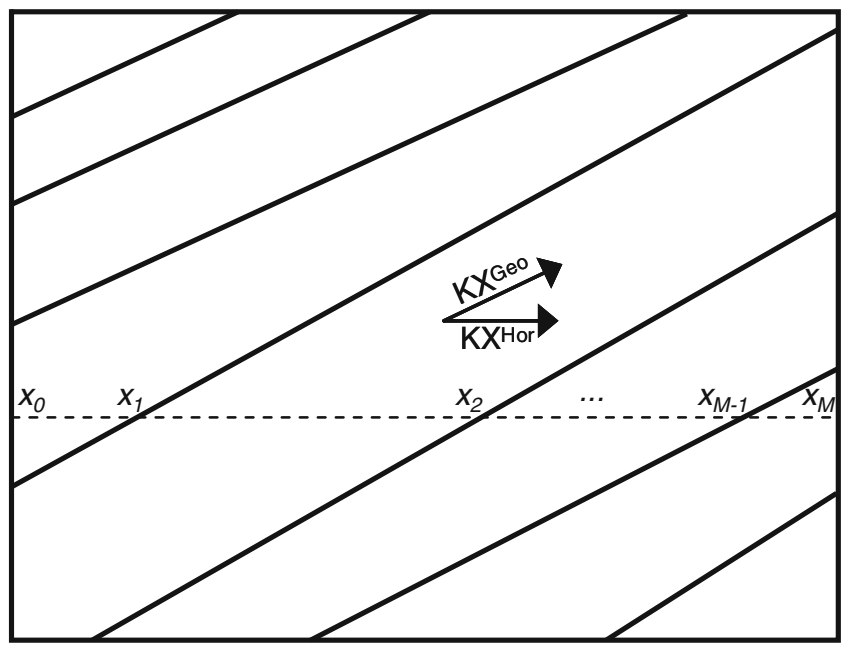

Fig. 8 Computing of horizontal permeability on a horizontal grid. Notation computational scheme: A popular scheme is based on the seven-point stencil and two-point flux approximation, i.e., the transmissibility $T X$ (in the $x$-direction) between cells $(i, j, K)$ and $(i+1, j, K)$ is computed from $x$-permeability values in those two cells. On a geogrid, TX will be computed from $K X^{\mathrm{Geo}}$, in agreement with flow in the layer direction. This preferred flow direction should be conserved when converting to a horizontal grid. This is actually an example of a case where the rescaled property is best described by full tensor permeabilities and multi-point flux approximation (MPFA): preserving the layer-aligned preferred flow direction from the geo-grid in a horizontal cells context can be achieved by including the non-diagonal permeability tensor terms, which contribute to noncell-aligned flow $[1,3,8]$. This approach was nevertheless rejected, mostly because full tensor permeabilities and MPFA are not available in most commercial simulators, but also because proper construction (averaging) of full tensors by rescaling from geo-cells to horizontal cells would require a significantly more elaborate procedure. We therefore developed a rescaling scheme within the framework of the seven-point stencil and two-point flux approximation, which enforces flow preference towards the original $K X^{\mathrm{Geo}}$-direction; intuitively by reducing the horizontal and increasing the vertical components of the permeability. We explain the calculation of $x$-permeability $K X$ in one horizontal cell. The first stage is to compute the horizontal projections of $K X^{\mathrm{Geo}}, K Y^{\mathrm{Geo}}$, and $K Z^{\mathrm{Geo}}$, for all layers $K_{1}$, $K_{2}, \ldots K_{N}$ (Fig. 7). Then the horizontal $x$-permeability in one of the geo-layers $\left(K X^{\mathrm{Hor}}\right)$ (Fig. 8) is the sum of the projections of $K X^{\mathrm{Geo}}, K Y^{\mathrm{Geo}}$, and $K Z^{\mathrm{Geo}}$ along the $x$-axis.

It is generally accepted that the appropriate permeability average for flow transverse to a permeability variation is the harmonic average, while the arithmetic average is appropriate for flow parallel to the variation [15]. Hence, we first compute the inline horizontal permeability as the harmonic average across the geolayers, along the dashed line in Fig. 8, where the $x_{m}$ are the geo-layer boundaries;

$$
\frac{x_{M}-x_{0}}{K X_{\text {inline }}^{\mathrm{Hor}}}=\int_{x_{0}}^{x_{M}} \frac{d x}{K X^{\mathrm{Hor}}(x)}=\sum_{m=1}^{M} \frac{x_{m}-x_{m-1}}{K X_{m}^{\mathrm{Hor}}}
$$

Then, the final horizontal cell $x$-permeability is computed as the arithmetic average of the inline $K X^{\text {Hor }}$ (volume-weighted sum over all present geo-layers). This scheme has intuitive appeal, as it will typically reduce the $x$-permeability and increase the $z$ permeability when mapped from geo-grid to hor-grid. Compared to the flow resulting from using direct 
averaging and standard transmissibility calculations on the horizontal grid, this scheme alters the flow-direction preference from horizontal to more diagonal, in agreement with the original layering, and should conserve preferred flow directions when converting petrophysics from the original geo-grid to the new hor-grid.

A straightforward alternative to the described rescaling algorithm would be to use averages of the appropriate geo-permeabilities directly; arithmetic averages for horizontal permeabilities and harmonic averages for vertical (denoted "standard averaging" in the following). In the grid construction software the user can select either standard averaging or the "flux-aligned upscaling" as described above (or any weighted average of the two methods). By geometry considerations the difference between standard averaging and flux-aligned upscaling increases with dip angle, i.e., the need for accurate upscaling is larger for large dip angles. An example of the impact on simulated fluid production is shown in Fig. 21 below.

\subsection{Inter-layer transmissibility multipliers}

Thin, low permeability barriers (e.g., shale) are often represented by vertical transmissibility multipliers in grids ("MULTZ" in ECLIPSE terminology [12]). During the conversion to horizontal layers each (geo)MULTZ is transformed to a set of MULTX, MULTY, and MULTZ parameters that cover the corresponding area in the horizontal grid, assuring that continuity is not lost. The values of the multipliers are kept unchanged in the transformation. The (geo)MULTZ is typically defined across a bed-aligned surface which is transformed to a set of horizontal and vertical surfaces on the hor-grid, that approximates the original (geo)surface, and such that connectedness is preserved.

\subsection{Fault transmissibility multipliers}

As mentioned earlier, faults in the geo-grid are geometry discontinuities, most often accompanied with fault transmissibility multipliers to honor the fault volume permeability. The defined multipliers are mapped directly to the associated cells in the horizontal grid. Hence, a fault in the horizontal grid will be geometry continuous (as the grid is completely regular), but the flow restrictions will be taken care of by parameter discontinuities and transmissibility multipliers (ref. Fig. 6).

Both inter-layer and fault transmissibility multipliers are created automatically by the conversion software.

\section{The basic simulation models}

To get as reliable results as possible from the base models, relatively fine meshes were used, with horizontal cell diameters $(\Delta x, \Delta y)$ of $40-50 \mathrm{~m}$, resulting in $40 \times 40$ cells in each layer. The basic geo-grid was also used as input to the hor-grid construction software, and was closely related to the structural geo-model for the segment, with 56 layers. Total model thickness is about $200 \mathrm{~m}$, whereof the gas cap is about $40 \mathrm{~m}$ thick, the oil zone is $13 \mathrm{~m}$, and the remaining $\sim 160 \mathrm{~m}$ is water.

For petrophysics and fluid data representative Troll data have been used. PVT-data are shown in Table 2 (only the end point values for the smooth oil and gas parameter curves are given).

Densities at surface conditions $\left(\mathrm{kg} / \mathrm{m}^{3}\right)$ :

$\rho_{\mathrm{w}}=1045, \rho_{\mathrm{o}}=890, \rho_{\mathrm{g}}=0.78$

Initial pressure is 158.2 bar at the GOC $(1,544.5 \mathrm{~m}$ sub mean sea level).

Three-phase relative permeability curves are shown in Fig. 9. Capillary pressure has been neglected. Initialization and fluid-in-place calculations were performed as described in Section 6.

Wells The wells in the segment were defined mainly in agreement with the strategy used on Troll. The horizontal oil producers were defined as true horizontal, $0.5 \mathrm{~m}$ above the OWC. All were placed with completions in a high permeability C-sand, and preferably below a shielding M-sand to prevent immediate gas breakthrough. For pressure support, four vertical waterinjectors were placed in the corners of the grid, injecting into the lower parts of the reservoir, and a horizontal gas injector parallel to the SW edge near the top (layer 2).

The well positions are shown in Fig. 10, which is a horizontal slice through the grid at producer-depth (the gas injector and water injectors are at different depths from this slice). Also shown is the permeability distribution at producer depth.

Table 2 PVT-data for Troll Segment model ( $B_{l}$ is volume factor, $\mu_{l}$ viscosity)

\begin{tabular}{lll}
\hline & Pressure 80 bar & Pressure 158.2 bar \\
\hline$B_{w}$ & & 1.017 \\
$B_{o}$ & 1.091 & 1.154 \\
$B_{g}$ & 0.0138 & 0.0068 \\
$\mu_{w}, \mathrm{cP}$ & & 0.45 \\
$\mu_{o}, \mathrm{cP}$ & 2.504 & 1.747 \\
$\mu_{g}, \mathrm{cP}$ & 0.0144 & 0.0173 \\
\hline
\end{tabular}


Fig. 9 Three-phase relative permeability curves used in the segment model
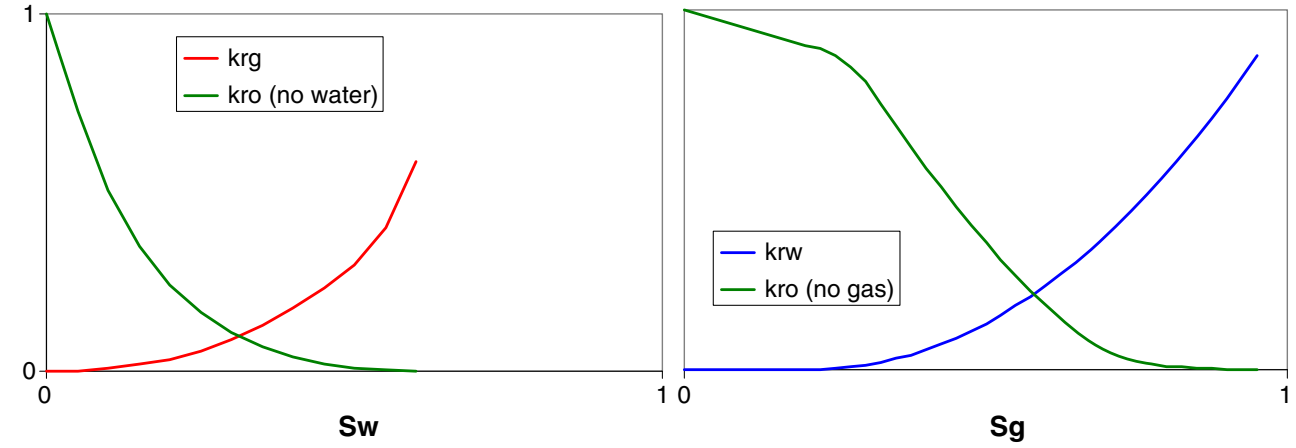

The way the producers are defined implies that we can have a physical expectation of how the gas production should be in each of the wells. As all the completions are at the same depth in a fairly homogeneous sand, which extends upwards to the gas zone parallel to the well, the gas breakthrough should be expected to be almost simultaneous in all the completions, and after breakthrough the gas production should have a steep increase.

In addition to the base well position scenario, some other cases with alternative well placements were also simulated. The results from these runs were consistent with those from the base cases, although not always as clear, mainly due to less predictable gas behavior.

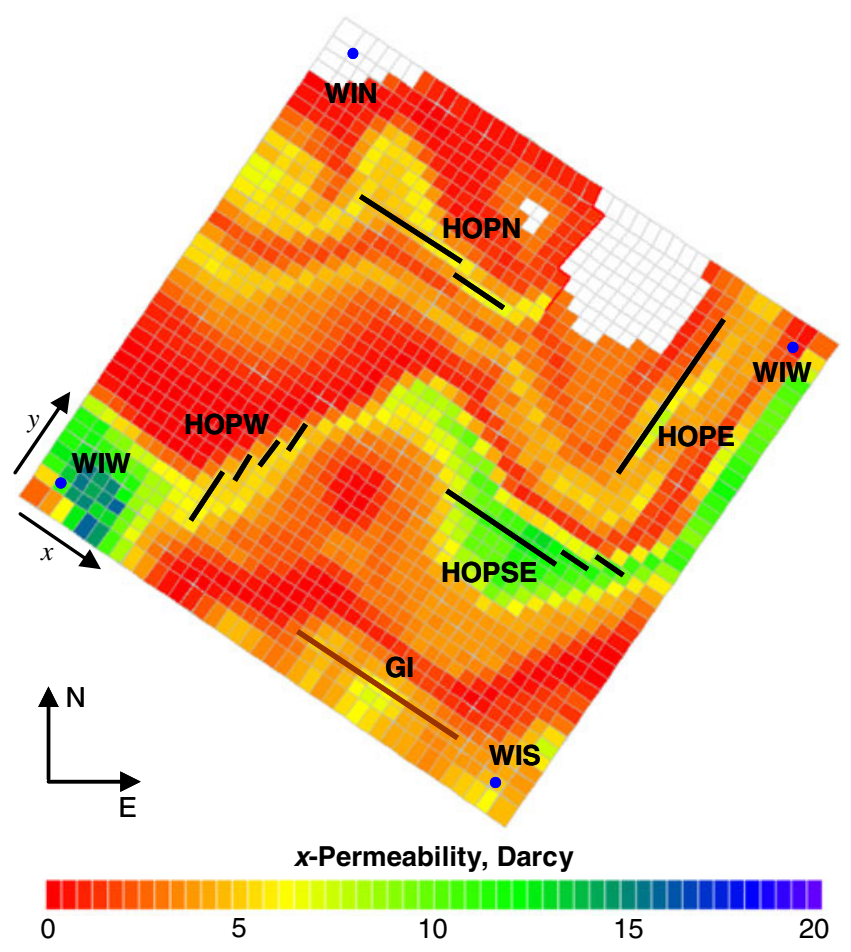

Fig. 10 Grid slice at producer depth, showing all wells
The producing and injecting target rates were set such that actual pressure depletion rate of the field was (coarsely) reproduced. Once appropriate target rates had been determined, this schedule was kept unchanged in all runs. The producers were defined with liquid rate as primary target, with target rate $1800 \mathrm{Sm}^{3} / \mathrm{D}$, constrained by maximum oil and gas rates of 900 and 70,000 $\mathrm{Sm}^{3} / \mathrm{D}$, and a minimum bottomhole pressure of 80 bar. Simulated total (field) oil production rate is shown in Fig. 11 for the three base cases described below. With exception of small irregular oscillations, the curves are essentially identical.

Time stepping and convergence control ECLIPSE uses an implicit numerical scheme where the non-linear equations are solved by the Newton-Raphson method, while the linear system is solved by Conjugate Gradients/Orthomin [12]. Through trial and error the time stepping scheme in each run was determined such that the run would complete without any non-linear iteration convergence failures while using as large steps as possible at any (simulated) time. For some of the runs time step sizes of less than one day were required. Still

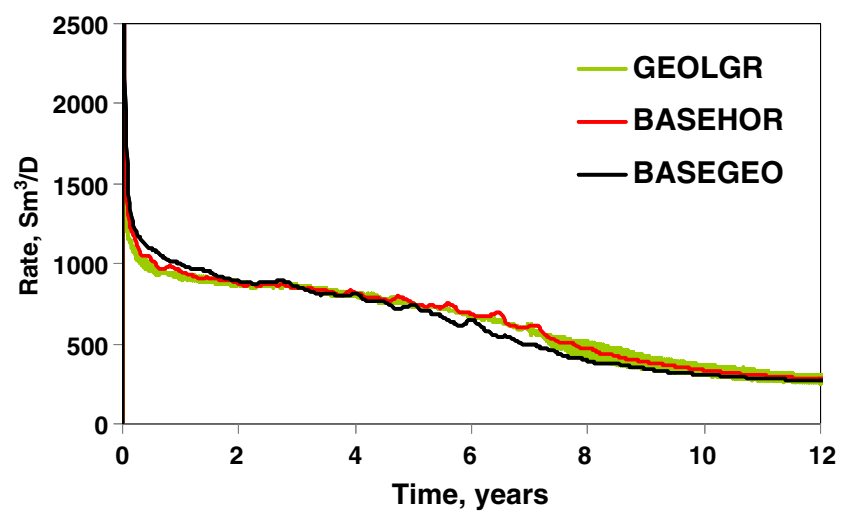

Fig. 11 Simulated field oil production rate for the three different base models 
the requirement of no non-linear convergence failures was only almost fulfilled.

\subsection{Base model 1: BASEGEO}

In the basic geo-grid, denoted BASEGEO, the layering was taken directly from the structural geo-model for the segment, with 56 layers. The grid comprises $40 \times 40 \times$ 56 cells ( 89600 total, of which 81403 are active). NNCs $(2,270)$ are present (see Figs. 2 and 5 for examples of cross-sections). Cells which are less than $0.1 \mathrm{~m}$ thick have been set inactive.

In a finite mesh any well completion in a cell volume is always interpreted as being at the cell center. Hence, each horizontal well completion was defined in the cell with correct $(i, j)$-index with center depth closest to the actual completion depth. The water injectors were completed in all layers in the appropriate depth range.

\subsection{Base model 2: BASEHOR}

The basic horizontal grid, BASEHOR, uses the same set of coordinate lines as BASEGEO, hence the cell resolution is unchanged in the $(x, y)$-plane.

In many of the horizontal grids that were constructed in this study the layer thicknesses $\Delta Z$ were defined by a geometric increase factor $m$. Typically $\Delta Z$ will increase from the GOC upwards and from the OWC downwards, such that if a layer has thickness $\Delta Z$, the layer above (gas) or below (water) will have thickness $m \cdot \Delta Z$.

The horizontal grid definition used in the BASEHOR model was,

- The oil zone, 13 layers, each $1 \mathrm{~m}$ thick

- From GOC upwards 8 horizontal layers were defined, with geometric increase factor $m=1.25$. The total thickness of the horizontal layers in the gas cap is $24.5 \mathrm{~m}$.

- From OWC downwards 10 horizontal layers were defined, with $m=1.25$. Total thickness of the water zone horizontal grid is $41.5 \mathrm{~m}$.

- Above and below the horizontal grid interval, the geo-layers from the BASEGEO model have been kept, with a total of 10 geo-layers in the top of the reservoir, and 35 geo-layers at the base.

Summing up grid definition top-down:

- Top $18 \mathrm{~m}, 10$ geo-layers

- Next 79 m, 31 horizontal layers

- Bottom 124 m, 35 geo-layers

- 76 layers in total
- Number of cells, 121,600

- Number of active cells, 63,774

- Number of NNCs, 865

Ref. Figs. 4, 6, and 10.

Petrophysics, regions and fault multipliers were resampled to the horizontal grid as described in Section 3.

The water and gas injectors were defined to match the perforation positions from the BASEGEO model as closely as possible, while all the horizontal producers were defined at their true depth.

In this study, all the horizontal wells are truly horizontal, such that a horizontal grid can honor completion depth exactly. For a general horizontal well with an undulating well path, the grid should be adjusted accordingly such that all perforations are at cell centers.

\subsection{Base model 3: GEOLGR}

Intuitively, and by experience from previous Troll simulation models, near-well simulated flow and production can be improved by using local grid refinement in the vicinity of the horizontal producers. For comparison, the case GEOLGR was built as a local grid refinement extension to the BASEGEO case. In the GEOLGR grid, a volume surrounding each well path has been defined with local refined cells (each coarse cell comprises $3 \times 3 \times 3$ LGR cells),

- Areally, the LGR was defined on a box which included the well path and one extra row of coarse cells on each side of the (coarse) well cells.

- Vertically, the layers to refine were chosen by a similar criterion, the LGR should cover all layers which contained perforations, and an extra coarse layer above and below.

- Total number of active global cells, 81,344

- Total number of active local cells, 129,336

- Total number of active cells, 210,680

- Number of NNCs, 2270

\section{Run series 1: hor-grid vs. geo-grid}

The purpose of the first series of runs was to compare the results from the different gridding scenarios (BASEGEO, BASEHOR, and GEOLGR). Naturally, we don't have available "correct" results with which to compare, so we need to use other criteria to assess the quality.

Firstly, the way the wells have been defined, all producers are parallel to the GOC. Moreover, all producers were placed in high permeability sands, such that the sand layer extends all the way to the GOC as 
a clean, homogeneous high-conductive sand, i.e. for a given well, the distance up to the GOC, measured along the high-permeable sand is identical for all perforations. Hence, we should expect simultaneous gas breakthrough in all perforations, and after breakthrough the gas-oil-ratio should rise quickly to its maximum value. Note however that wells HOPN and HOPE has no or only a small gas cap above, so gas production from these wells should be expected to be less predictable, since it will be caused by a more complex gas flow pattern.

Secondly, we will use a standard iterative approach; when we change the data deck only incrementally in a manner that is obviously an improvement, we expect an incremental improvement in the solution. This argument has however some limitations, as, e.g., when we reduce grid cell sizes, an improved solution is intuitively expected. On the other hand this can lead to reduced numerical accuracy, so the net gain/loss can be difficult to identify.

A number of initial runs were performed to test various scenarios, for sensitivity studies, and to gain knowledge of the general behavior. Following this trial stage an extensive test scheme was set up. From this scheme, only a few figures documenting key results will be reported. Note that the conclusions which are stated in the text are typically based on observed behavior from a large number of runs, although only an excerpt of these runs are described by text or figures in the paper.

Field (total) production results Simulated oil production was as good as equal in the three base cases (taking account of numerical accuracy), Fig. 11. Water rates differed in that the small fall-off occurred earlier in the BASEGEO model than the other two cases (Fig. 12).

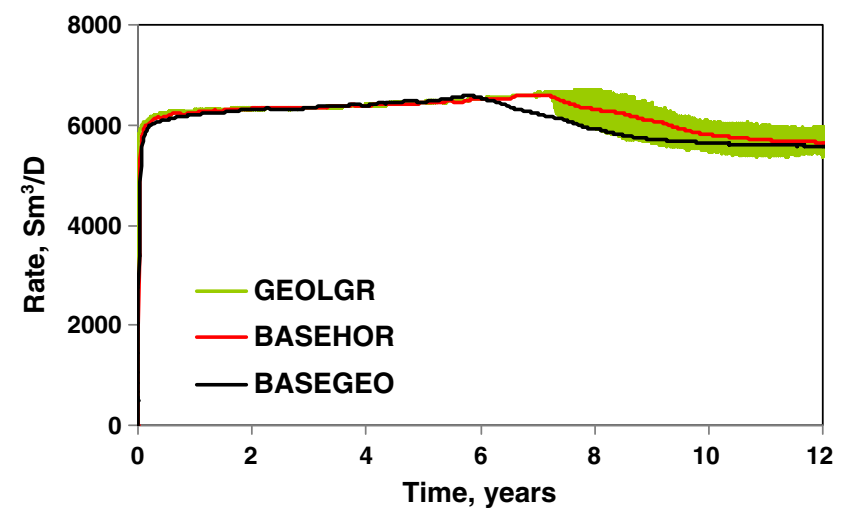

Fig. 12 Simulated field water production from the three base case models

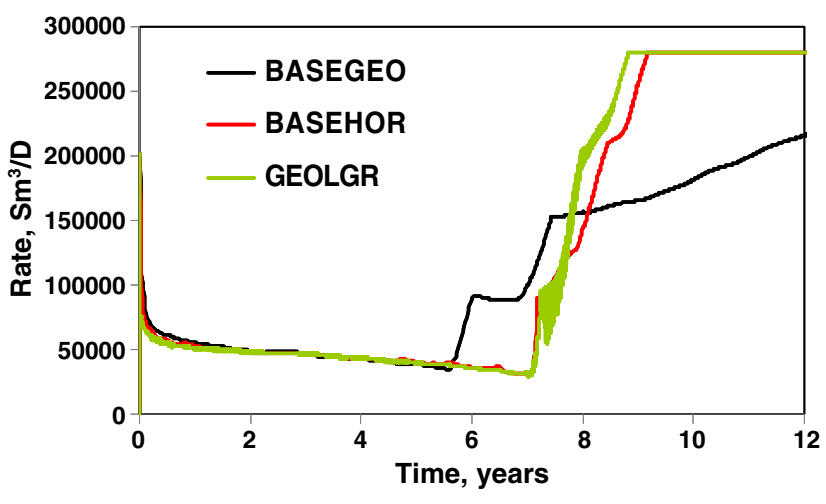

Fig. 13 Simulated field gas production rate from the three base cases

The total (cumulative) produced water was for practical purposes equal.

The largest differences were seen in the gas production, Figs. 13 and 14. The BASEGEO case is here significantly different from the other two cases. Also note the rate oscillations for the GEOLGR case. These are a result of the scheme to distribute rates between phases and wells which is used by ECLIPSE to honor rate targets and constraints. Especially for problems which require a large number of iterations to converge the linear system, distribution of phase production can be erroneous. In most cases this "feature" is not significant, but it appears to be especially noticeable in large models which contain LGRs, probably due to the message-passing between the global and local grid solvers (material balance and rate/phase totals are nevertheless always conserved).

Reservoir pressure is very sensitive to the gas production, but the depletion rate was preserved as good as possible between runs. The pressure trend differs somewhat between the cases after gas breakthrough, with

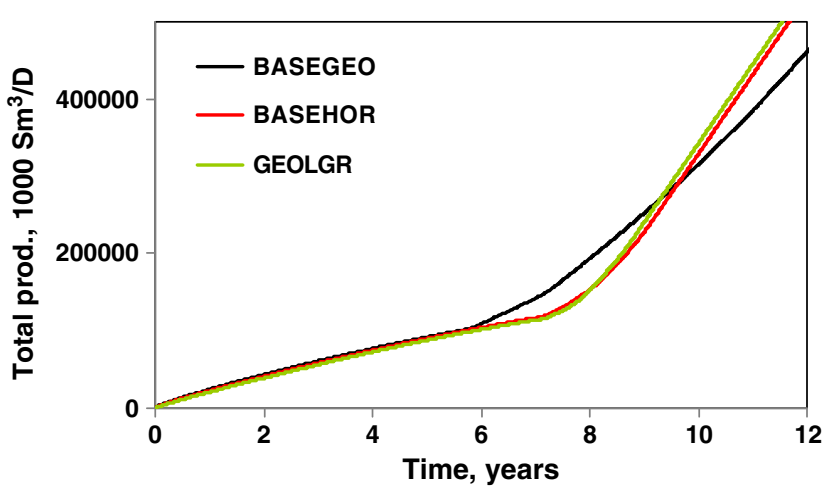

Fig. 14 Simulated field cumulative gas production from the three base cases 
BASEGEO following a different trend than GEOLGR and BASEHOR, which were relatively equal.

Well production results Oil rates, and especially cumulative production were comparable in the three cases. In general, where (slight) differences were present, the BASEGEO case was the deviating case, while BASEHOR and GEOLGR were relatively equal. The same was observed also for water rates and water production, with BASEGEO differing more significantly from the other two cases.

As expected, the largest differences between the three cases were seen for well gas rates. The behavior seen in Fig. 15 (gas rate from well HOPW) is representative for all the wells. As mentioned earlier, by the model set-up the gas rate should have an abrupt and considerable rise after gas break-through. This has been captured by the BASEHOR and GEOLGR cases, but not by the BASEGEO. Generally, in all results concerning gas production from wells the BASEHOR and GEOLGR cases were similar or equal, while the BASEGEO case deviated significantly, and was also not in agreement with the expected qualitative production profiles mentioned above.

Fluid contact movement By comparing the fluid front movement near the contacts (especially the GOC) in a 3-D graphics program it was found that apart from the difference in resolution, the three cases in question had relatively equal behavior. To test this further, some LGR-cases were constructed from the BASEGEO model with local grid refinement near the initial fluid contacts, in the upper parts of the oil zone, or covering most of the oil zone and gas cap (it was essential not to use LGR in the vicinity of the horizontal wells in this part-study). The results from these runs were qualitatively equal to the BASEGEO-results, and differed noticeably from BASEHOR and GEOLGR. Hence,

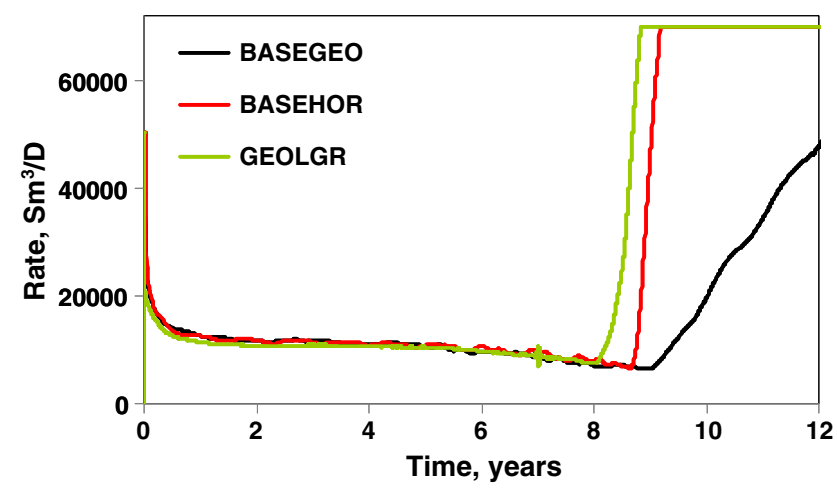

Fig. 15 Gas rates from well HOPW for the three base cases insufficient resolution in the contact movement zone cannot explain the differences between BASEGEO and BASEHOR/GEOLGR.

\subsection{Observations from the comparison runs}

BASEGEO was unable to capture the qualitative shape of the gas production as expected from the physics, while BASEHOR and GEOLGR both appeared to succeed in this respect. As the two runs also generally were in agreement, it is tempting to conclude that they are closer to reality than BASEGEO. The differences are not tied to fluid contact movement, but rather to the well modeling. It is therefore of interest to address this topic closer.

\subsection{Completion modeling}

In a simulation grid well perforations cannot always be defined at their exact position, but are represented at the cell centre of the grid cell which contains them. In this context it is apparent that due to the short distance from the fluid contacts to the perforations, a small error in perforation depth may have large consequences. As the differences in, e.g., gas rates as seen in Fig. 15 could not be explained by the simulated fluid contact movement it is natural to investigate the significance of the perforation modeling.

Figure 16 is a schematic of how the perforation depths in well HOPSE were represented on the grid. As an example, regard the cell which contains the perforation closest to the heel. At the outset all cells containing the perforation's $(x, y)$-coordinates are candidates for being a completion cell. The exact perforation depth was $1,556 \mathrm{~m}$, and among the candidate cells the one with center depth at $1,556.4 \mathrm{~m}$ was closest to correct

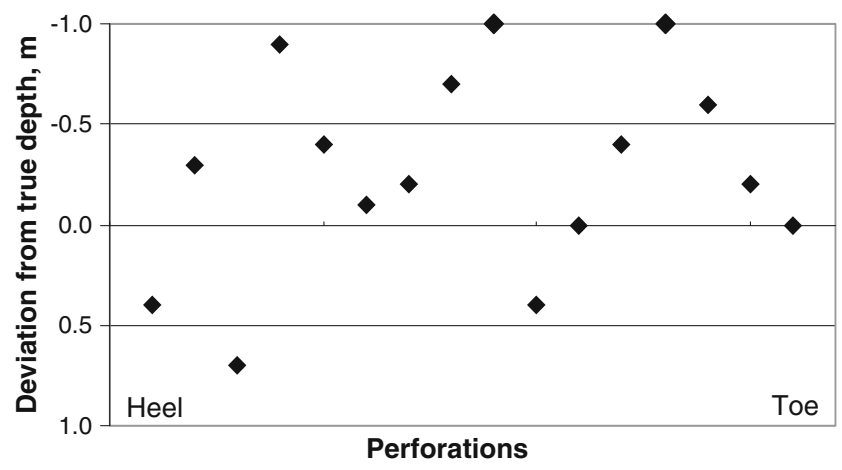

Fig. 16 Difference between modeled (cell centers) and true perforation depths, case BASEGEO, well HOPSE 
depth. Hence the perforation in question was modeled as being at depth $1,556.4 \mathrm{~m}$ on the grid, and the error is $0.4 \mathrm{~m}$-the leftmost point in the figure. Note that the deviations are not large; on this grid the error was in the range $\pm 1 \mathrm{~m}$.

To test if such small deviations can have noticeable influence some sensitivity cases of BASEGEO were run: some of the perforations with the largest deviations were moved one cell downwards. Shifting the modeled perforation depth in this manner had large impact on the results; as an example it was seen that a change in modeled perforation depth by only $0.5 \mathrm{~m}$ in a few perforations delayed simulated gas break through by about one year. Hence, accurate modeling of completion depths appears to be a significant factor. This issue will be discussed further in Section 6 below.

\subsection{Summary of series 1 runs}

- Simulated fluid frontal movement was qualitatively equal in all three base models (BASEGEO, BASEHOR, GEOLGR)

- The BASEGEO model failed to capture essentials of gas flow near the producing wells. To a lesser degree the same concerns water flow

- The GEOLGR and BASEHOR models were able to capture much of the same features with comparable results. Simulated results were more in accordance with physical expectations than BASEGEO

- One benefit of the BASEHOR model compared to BASEGEO is that the high resolution cells can be concentrated to the domains where they are most needed

- The GEOLGR model suffers from partly severe rate oscillations due to the handling of well constraints, but the moving average is probably reasonably accurate.

The key observations from this series of runs are that representation of completion positions has great influence, and that the most sensitive parameter is gas production (see also Section 6), i.e., accurate modeling of the interaction between the dynamic GOC and the well completions should receive primary attention in studies of this kind. As this interaction will be stronger when the height of the vertical interaction zone is reduced, accurate modeling of fluid contacts and completions becomes increasingly significant when the thickness of the oil-leg is reduced.

For this particular study the simulated near-well fluid flow and gas production was in better agreement with physical expectations with the horizontal grid model than the traditional geo-grid model. Further, results for other not-so-sensitive parameters like oil and water production, were comparable for the two models, indicating that the horizontal grid was able to capture essentials of general flow, in spite of the lack of alignment to geology.

Based on these results, and also results from other projects using horizontal grids (with a range of oil leg thicknesses and different kinds of heterogeneity), we can conclude that this kind of grid can be a viable alternative in many settings.

By comparing computing times, another advantage of the horizontal grid is revealed.

Computing times (CPU) for the three base cases:

\begin{tabular}{lr}
\hline BASEGEO & $183 \mathrm{~min}$ \\
BASEHOR & $133 \mathrm{~min}$ \\
GEOLGR & $2395 \mathrm{~min}$ \\
\hline
\end{tabular}

Hence, although the results obtained from the BASEHOR and GEOLGR cases were almost identical (disregarding the oscillations), the local gridrefinement case required 18 times as long computing time as BASEHOR.

\section{Grid sensitivity test}

The main purpose of the presented study was to support the Troll project in determining the strategy for building the next generation oil zone simulation models. As such the main focus was naturally on representative models for Troll. However, some questions raised above motivated a separate study where the role of accurate perforation depths and fluid contact description were directly addressed by a sensitivity study on grid block sizes. To that extent, a simple generic model was built, measuring $1,485 \times 675 \times 157 \mathrm{~m}(x, y, z)$, with a constant slope angle of $7^{\circ}$, and with highest point $1,410 \mathrm{~m}$ below surface at the eastern edge. The oil zone was $12 \mathrm{~m}$ thick, between 1,538 and 1,550 $\mathrm{m}$.

Model setup The petrophysics was modeled with layer-constant properties in 20 layers, alternating between high-, medium-, and low permeability zones. Fluid properties were intentionally chosen significantly different from the Troll study, to make the resemblance to the already studied model as small as possible. For the same reason, the single horizontal producer was positioned so that it traversed almost all of the layers, in contrast to the well positions in the Troll model. The model dimensions and grid cell sizes were chosen so that the well could be represented in the same position on all the grids. The coarsest grid had cell diameters 
of $135 \mathrm{~m}$ (identical in $x$ - and $y$-directions), while the refined grids had cell diameters of $45,27,15$, and $9 \mathrm{~m}$, respectively. In the vertical direction the 20 different layers defining the petrophysics were used directly for the coarsest grid, and grids with 32, 49, 96, and 125 layers defined the refined cases. The horizontal well was completed at constant depth $0.6 \mathrm{~m}$ above the OWC, and produced at a constant liquid rate of $700 \mathrm{Sm}^{3} / \mathrm{D}$, with a minimum bottom hole pressure 130 bars below initial pressure.

The corresponding horizontal grid models were hybrid models with ten layers in the oil zone, and four horizontal layers above the GOC and below the OWC, using the coarsest geo-grid layering in the upper and lower parts.

Initialization and initial oil in place The models were initialized to equilibrium, with initial fluid content in each cell determined by the cell center saturation. Alternative schemes based on volume-averaged saturations may be better with respect to initial fluidin-place calculations, but has the drawback that the initial fluid distribution is generally not in equilibrium. The volume-averaged scheme was nevertheless tested for some cases, and results were far from the general trend-providing an additional argument for rejecting this method.

In the horizontal grids, the fluid contacts are accurate, and calculation of cell pore volumes straightforward; hence the oil-in-place will be accurately computed in the hor-grid models. Using the reported oil-in-place (OIP) from the finest hor-grid model as reference, the OIP in the geo-grid models deviated by about $10 \%$ in the 135 m-grids. For the finer grids, the deviation was not surprisingly dependent on the vertical refinement. In general, the models with grid diameters of $45 \mathrm{~m}$ and less, and with at least 32 layers, had an OIP-deviation of less than 3\% compared to the reference. The variation between the four different horgrids was less than $0.1 \%$.

Test scheme The planned test scheme was to run simulations using successively refined geo-grids, to see if this series converged in some sense as grid cell volumes approach zero; and if a limit could be identified, compare it to the hor-grid simulations. The practical difficulty in carrying through this scheme was that the runs with the finest grids had extremely bad convergence properties, and some had to be terminated due to unacceptable long run times. However a pattern emerged that allowed us to draw relatively clear conclusions independent of the results that were never achieved. Results will be presented below according to the "revised" test scheme.

The coarsest vertical model in the test scheme (with 20-layers and layer thicknesses between 3 and $10 \mathrm{~m}$ ) proved completely useless, and will not be discussed any further. The vertical resolution was simply too coarse. It should be noted that this model had grid cell dimensions that are "typical" in simulation studies, but still grossly insufficient for thin oil-zone problems.

\subsection{Perforation depth modeling errors}

It was noted above that the position of a well perforation in a grid cell will always be approximated by the cell center position, which generally is an error in positioning, and can imply erroneous simulated fluid production. For thin oil-zone problems the perforation depth error is the relevant parameter. When defining perforation cells we are hence forced to choose how to approximate actual perforation depths. To test what impact the errors in perforation depth have on simulated flow and production three cases where defined, using the geo-grid with cell diameter $27 \mathrm{~m}$ and 32 layers. The cases had different but valid strategies for selecting perforation cells. In each column the completion cell was chosen according to its center depth, as:

(a) Closest to true depth, but in the oil leg ("High")

(b) Closest to true depth ("Nearest")

(c) Closest to but not above true depth ("Low")

These runs were compared to the corresponding horgrid run.

Results for simulated oil and gas rates are shown in Figs. 17 and 18.

If our assumptions regarding perforation depths are valid we should expect: As the perforations in the "High" case are closest to the GOC, this case should have the earliest gas breakthrough, followed by the "Nearest" case, which has some perforations that are too high, but the highest have been excluded. In the "Low" case all perforations are too deep, hence should have the latest gas break through. The hor-grid case has correct perforation depths and should fall in somewhere between these.

For water production the opposite should be expected, with highest water production in the "Low" case (recall that the sum of oil and water rates is constant).

The gas production curves (Fig. 18) are in agreement with the expectations, with the "Nearest" and hor-grid results sandwiched in between the "High" and "Low". Results for oil production (Fig 17) also mainly agree with the expectations, but are less conclusive than the 


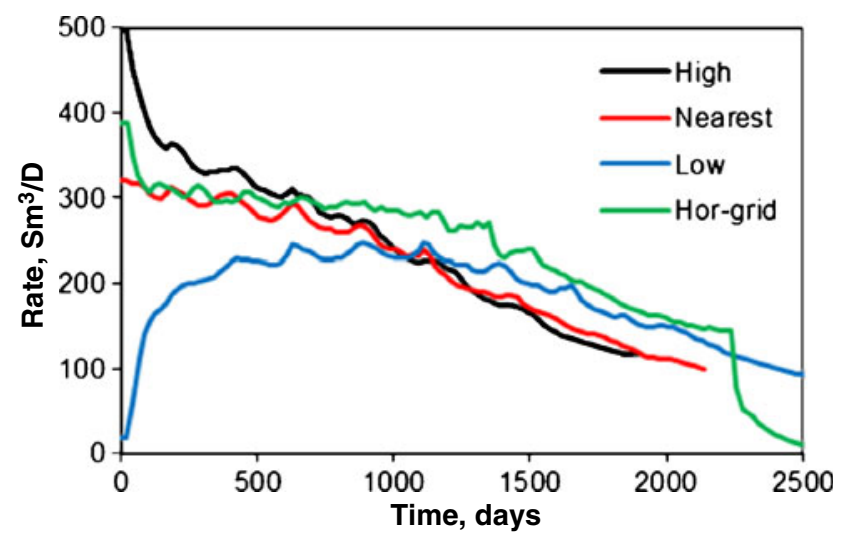

Fig. 17 Single well model, oil rates: Significance of well perforation approximation; modeled at base of oil zone ("High"), closest to actual perforation depth ("Nearest"), and at top of water zone ("Low"). Cell diameter 27 m, 32 layer case. Corresponding horgrid curve shown for comparison.

gas curves (e.g., the high oil rate in the "Low" case is due to the OWC contact moving downwards during production, which complicates the comparison) This is consistent with our findings from Section 5, namely that as gas is more mobile than oil and water, the simulated gas production is more sensitive to the modeling strategy than the other two phases.

One interesting finding from this case study is the relatively large difference between the runs, as all of these could be regarded as valid approximations, depending on the context. The conclusion is that the perforation depth error (which will always be present on geo-grids) can have relatively large influence on simulated production.

\subsection{Areal grid refinement}

The maximum perforation error for a completion in a layer of thickness $D Z$ is $0.5 D Z$. The way we constructed

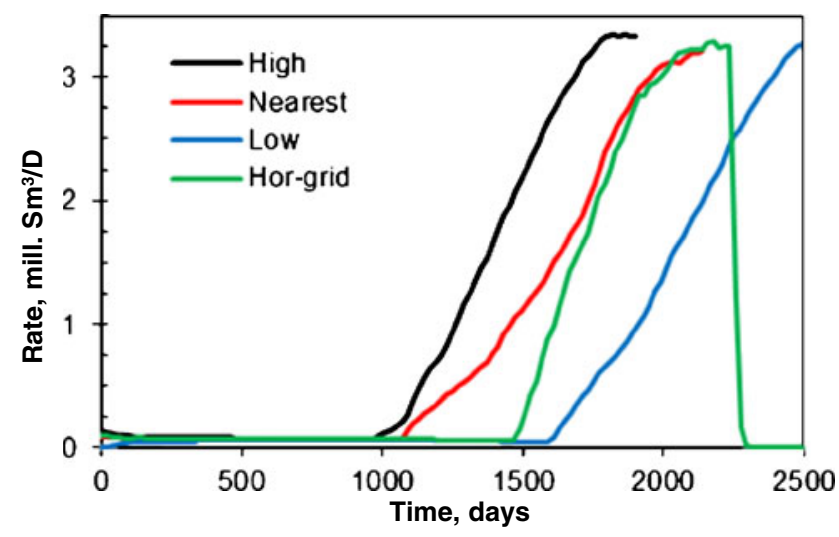

Fig. 18 Single well model, gas rates. Sensitivity as in Fig. 17

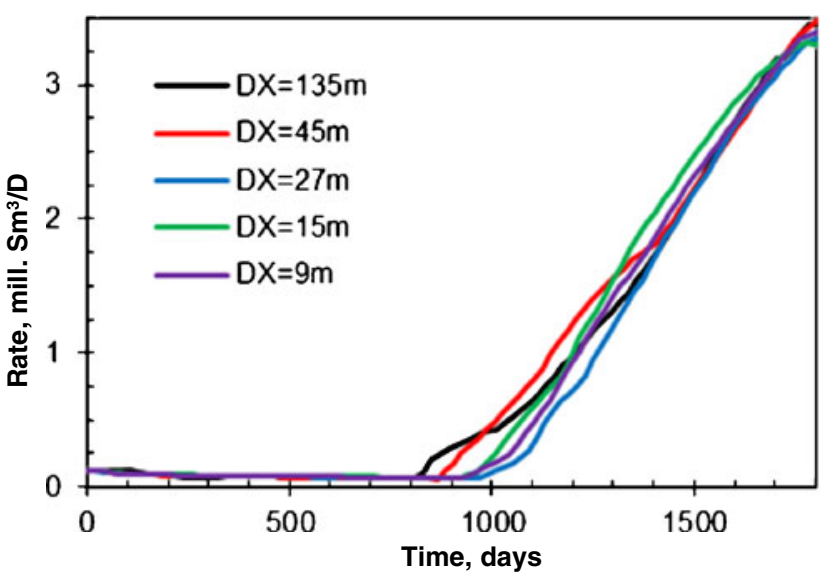

Fig. 19 Single well model, gas rates. Variation of cell diameter DX. 32 layers

the areal grid refinements was by subdividing all cells in a coarser grid into $3 \times 3$ or $5 \times 5$ cells. In that way the $(x, y)$-positions of all coarse grid cell centers are preserved in the refined grid. Hence the perforation error is not reduced by areal grid refinement; on the contrary it will probably increase as new perforation cells are introduced.

On this background, if perforation depth error is a significant factor to the total simulation error in thin oil-zone problems, we cannot expect that the error will be noticeably reduced by areal grid refinement.

In this series of runs cell diameters were reduced while keeping the vertical definition unchanged. The test was performed using the geo-grid with 32 layers, where layer thicknesses are 3 to $5 \mathrm{~m}$ (this was the finest vertical resolution that could be run for all of the cell diameters). The results for gas production rates are shown in Fig. 19 (oil and water rates differed less than the gas curves). The two coarsest grids (cell diameters 135 and $45 \mathrm{~m}$ ) deviate from the others, but not much. For cell diameters $27 \mathrm{~m}$ and less the results are as good as identical, and if the curves converge as cell diameters approach zero the "limit" has been reached already at $D X=27 \mathrm{~m}$. Whether this "limit" is an acceptable solution is still an open question, especially taking account of the perforation error that is present in all these runs.

\subsection{Vertical grid refinement}

The areal grid refinement test indicated that results did not change significantly when the areal resolution was reduced. Hence, we consider it as sufficient to use grids with a cell diameter of $45 \mathrm{~m}$ in the vertical refinement test (furthermore, smaller cell diameters could not be run for the finest vertical resolutions). 
The vertical resolutions of the grids in this series of runs were,

- 32 layers, thicknesses 3-5 m

- 49 layers, thicknesses 2-3 m

- 96 layers, thicknesses $1.17-1.5 \mathrm{~m}$

- 125 layers, thickness $1 \mathrm{~m}$

(the thicknesses are for layers including the oil zone. Some layers in the water zone were thicker.)

In this series the perforation depth error is reduced for each level of refinement, hence we expect total simulation error to be reduced as layer thicknesses approach zero.

Simulated results for oil and gas rates are shown in Figs. 20 and 21, where results from the hor-grid case with cell diameter $45 \mathrm{~m}$ have been included for comparison.

For the oil rate (Fig. 20) the differences are not large, but the curves do approach a limiting curve as $D Z$ approaches zero. The hor-grid curve is near this limiting curve, but still deviates noticeably from it. The gas rate curves (Fig. 21) are more conclusive. Both the time of gas break through and the rate increase with time clearly converge towards a limiting curve that should not be very different from the run with $n z=$ 125. The hor-grid curve is also near this limit curve, except at the highest gas rates. Note also that the curve " $n z=32$ " is the same as the " $D X=45 \mathrm{~m}$ "-curve in Fig. 19, confirming our suspicion that the curves had not converged.

The maximum perforation depth error is still $0.5 \mathrm{~m}$ in the finest of these cases, but the difference between the cases $n z=96$ and $n z=125$ is very small, indicating that they are approaching a "limit curve". The difference between the results from the finest geo-grid and the

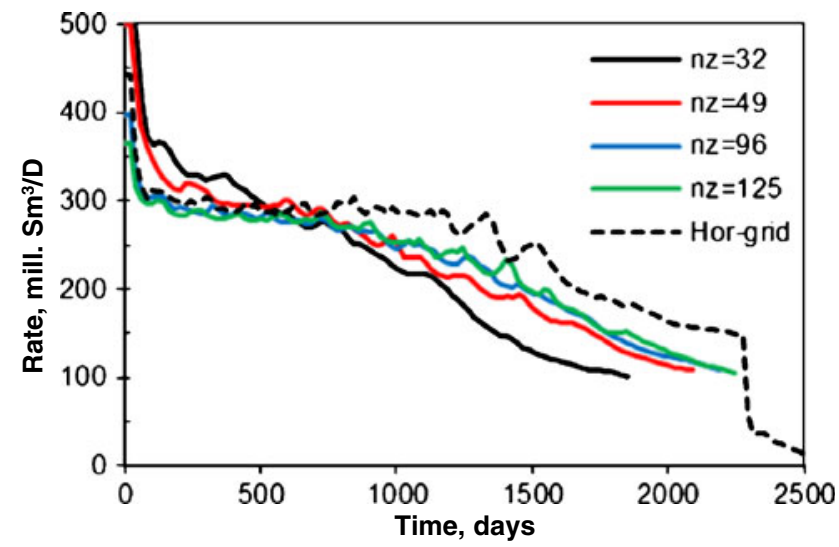

Fig. 20 Single well model, oil rates. Variation of vertical resolution ( $\mathrm{nz}=$ number of layers), cell diameter $=45 \mathrm{~m}$. Corresponding hor-grid curve shown for comparison

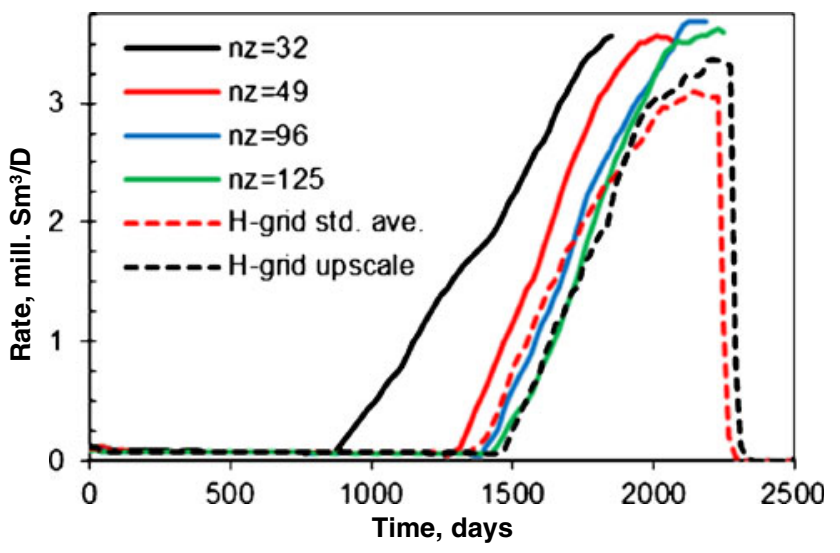

Fig. 21 Single well model, gas rates. Sensitivity as in Fig. 20. Also included: Result from hor-grid using standard averaged permeabilities

hor-grid can be explained partly by the still present geo-grid perforation error, partly by the non-horizontal representation of the fluid contacts on the geo-grids, and partly by the approximate representation of the petrophysics on the hor-grid.

However, the main observation from this test case is that the geo-grids do converge as layer thickness is reduced, and that the "limit curve" is not far from the hor-grid results. Hence the geo-grid and hor-grid simulations converge to almost the same results as cell sizes approach zero (possibly, even better convergence could have been achieved by repeating the vertical refinement test for smaller cell diameters. Such attempts were however unsuccessful due to convergence problems).

Hor-grid upscaling For comparison, one hor-grid run was done with the "standard averaging" scheme. The difference in simulated gas production can be seen in Fig. 21, where both "standard averaging" and "fluxaligned upscaling" have been included. For the present model with $7^{\circ} \mathrm{dip}$, the difference is noticeable, and the curve for flux-aligned upscaling is closest to the "limit curve" from the geo-grids, so the upscaling appears to have had the desired effect. Whether the difference is big enough to validate the need for the upscaling in this case is a subjective decision.

\subsection{Hor-grid sensitivities}

All the hor-grids were constructed from geo-grids with the coarsest layering (20 layers), and cell diameters 135, 45,27 , and $15 \mathrm{~m}$ were used in the hor-grids. Resulting gas rates are shown in Fig. 22. The case with the largest cell diameters $(135 \mathrm{~m})$ deviates a little, but the other 


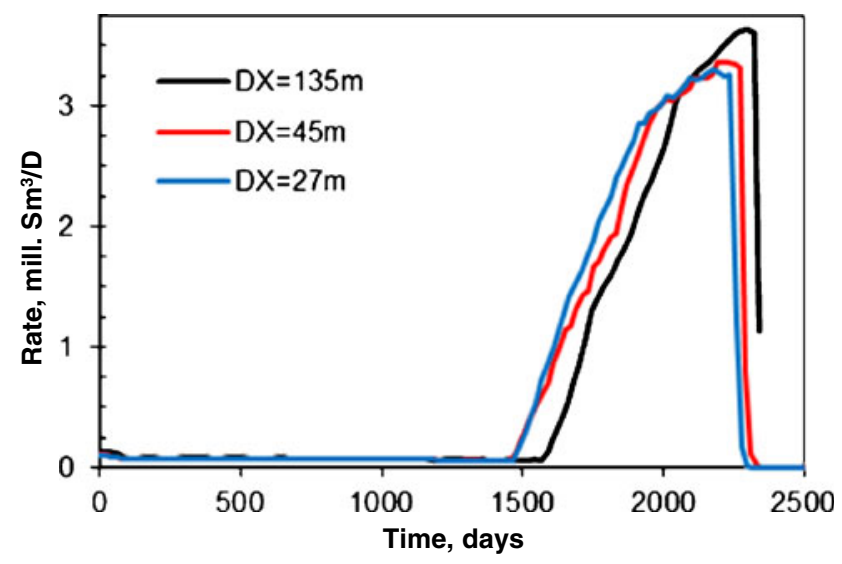

Fig. 22 Single well model, gas rates. Hor-grids with variation of cell diameter $\mathrm{DX}$. The curve for $\mathrm{DX}=15 \mathrm{~m}$ was identical to "DX $=27 \mathrm{~m} "$

cases were very similar (curves from the cases $D X=$ $15 \mathrm{~m}$ and $D X=27 \mathrm{~m}$ were identical).

For the grid with cell diameters $27 \mathrm{~m}$ hor-grids were also constructed from the geo-grids with 32 and 49 layers, hence generating hybrid grids with higher resolution in the upper and lower parts of the model. Results from these runs were identical to the comparison hor-grid results, supporting that high resolution is not imperative for flow computations in the one-phase regions.

The main conclusion from this test was that the horgrid simulations were not very sensitive to cell diameter variation; hence reasonably large cells can be used without significant loss of accuracy. This is in agreement with the results from Section 6.2, as perforation depth errors are absent on hor-grids.

\subsection{Summary of the grid sensitivity test}

In general the findings from this test agree with those from Section 5.3, and hence strengthen the conclusions,

- The overall quality of the simulated results are closely tied to the perforation depth errors, i.e. the maximum cell thickness for cells containing well completions

- The maximum perforation depth error is not reduced by reducing cell diameters

- Results of acceptable quality could be obtained on geo-grids, but required very thin (and hence very many) layers. The needed resolution was far finer than what would be possible in realistic-sized models, and the resulting convergence properties and computing time were not acceptable
- Results of comparable quality to the finest geo-grid results were obtained with hor-grids with acceptable cell sizes and computing times

- The hor-grid simulations were not very sensitive to areal resolution

Restricted to corner-point grids, the horizontal grid model (including hybrid grids) is an attractive method to handle the combined challenge of accurate fluid contact and well description. The negative aspect is obviously the lack of direct mapping to the geological features. We concluded above that the most critical factor in thin oil-zone problems was the representation of the well path, so the preferred approach would be to utilize a grid which could honor both geology and well paths. Unstructured grids, noticeably Voronoi- or PEBI-grids (see, e.g., [10,12] and the references herein) allow for accurate modeling of both geology and well paths, and as such appear to be ideal for handling of the problem in question. As an example, Mundal, Keilegavlen, and Aavatsmark [11] studied near-well flow on generalized grids in two dimensions. The approach appears promising, but so far successful use of unstructured grids has been limited to two-dimensional and small 3-D (actually 2.5-D) models. Hence, cornerpoint grids and equivalent schemes are still preferred for large models.

\section{Run series 2: grid coarsening}

The cell sizes chosen in the runs described in Section 5 were unrealistically small, to enable construction of a reliable reference case. For real-size or full field models cell sizes will be larger, and in this batch of runs the significance of cell size is investigated. As found in Section 6, simulations on geo-grids coarser than BASEGEO must be expected to be of limited value. Hence the coarsening test was performed with horizontal grids only.

Two new grids were constructed, with cell diameters twice and three times the GEOGRID diameters respectively. First new geo-grids with the updated cell sizes were made (keeping the original layering). Then new horizontal grids were constructed from these, using the same (horizontal) layering scheme as in the BASEHOR case.

Some results are shown in Figs. 23, 24, 25 and 26.

In general the differences between these three cases were noticeable, but not significantly large. Field production of all three phases and reservoir pressure are equal (within numerical accuracy) (Figs. 23, 24 and 25). At well level water production is almost identical, while 


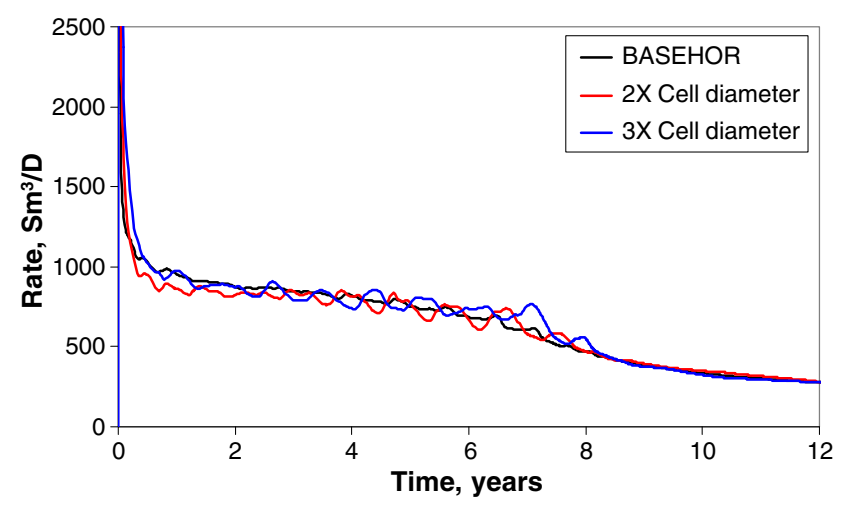

Fig. 23 Field oil rates; hor-grid with different cell sizes

there are some differences in gas production (Fig. 26). The $3 \times$ diameter case can in some sense be regarded as representing the point where coarsening effects start to be of significance; hence we can conclude that a cell diameter of $100-150 \mathrm{~m}$ is sufficiently fine to preserve accuracy in these models.

If results from the finest cell size (BASEHOR) are taken as most accurate, evidently some quality loss must be expected when the grid is coarsened (as observed). The cell size used in the BASEHOR model is too small to be used in a full-sized (field) model, so some loss in quality must be accepted. The interesting question in this context is how much of the quality loss is due to the coarsening compared to what must be ascribed to the conversion to horizontal cells. This is case-dependent, and no general recommendations can be given. As an example a concurrent study was done on the Gjøa Field, where the dip of the structure is larger than on Troll, and the layer thicknesses are generally smaller. As expected, in order to capture the permeability variation on a horizontal grid, cell sizes

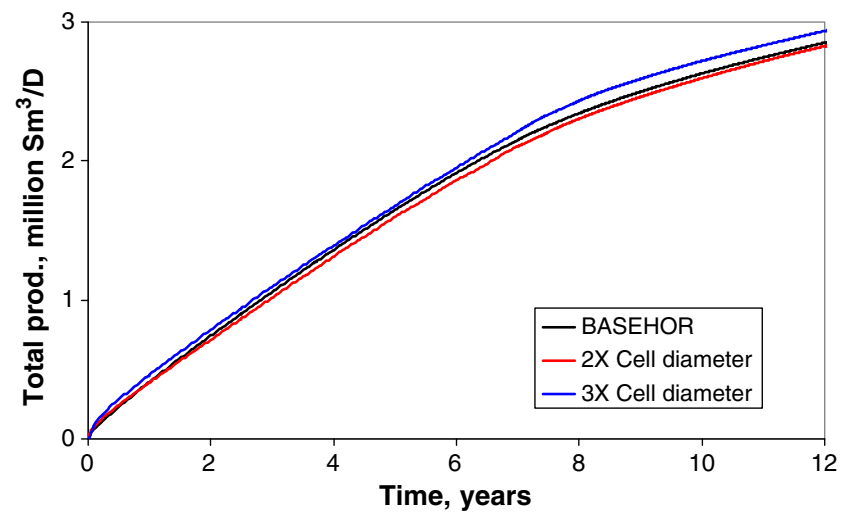

Fig. 24 Field total (cumulative) oil production; hor-grids with different cell sizes

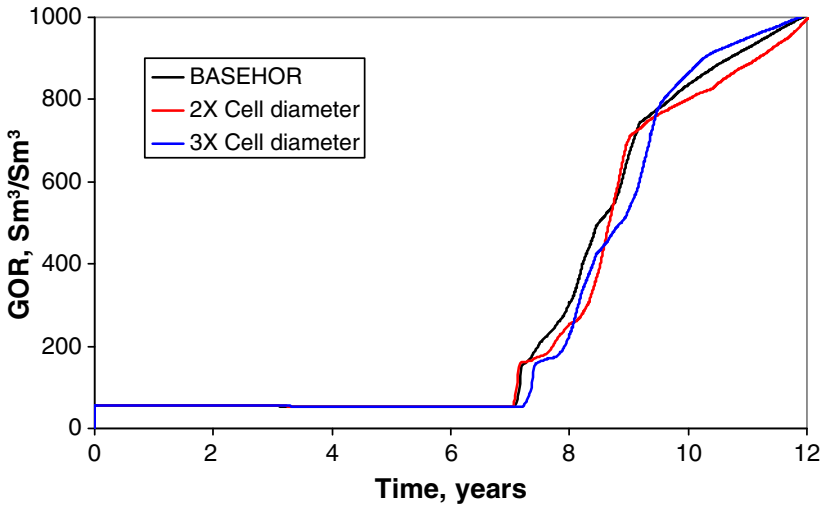

Fig. 25 Field gas-oil ratio; hor-grid with different cell sizes

had to be smaller (both horizontally and vertically) than the findings in the Troll project.

By the preceding arguments it appears to be sufficient and necessary to use high resolution cells in the dynamic domains (especially near wells), hence a tempting approach could be to use a relatively coarse hor-grid model with LGRs in chosen areas. Such grids are intuitively attractive (probably optimal), but have not been studied in this paper.

CPU times for the three levels of coarsening:

\begin{tabular}{lc}
\hline BASEHOR & 133 min \\
HOR 2X Cell diameter & 8 min 34 s \\
HOR 3X Cell diameter & $2 \min 34 \mathrm{~s}$ \\
\hline
\end{tabular}

For this model the results obtained from $2 \times$ cell diameter were acceptable, and possibly also the $3 \times$ diameter case. Hence, computing times can be significantly reduced by using coarser cells, a conclusion that is relevant and more important when adapting the technique to larger models.

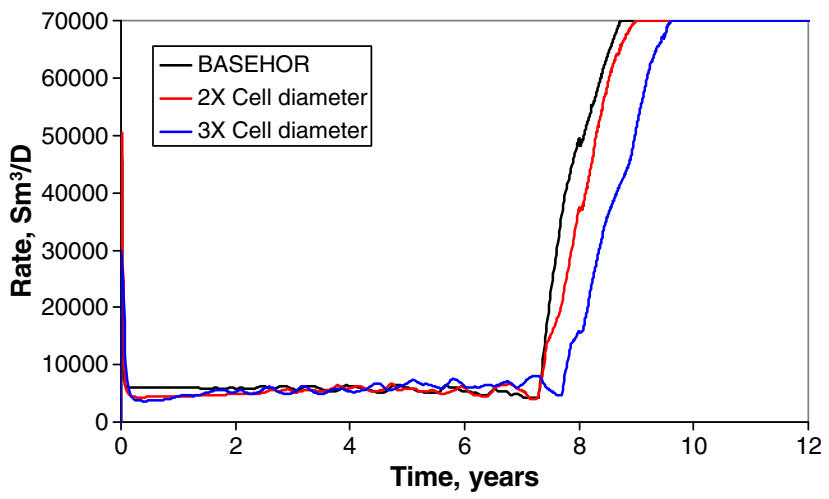

Fig. 26 Gas production rates in well HOPE; hor-grids with different cell sizes 


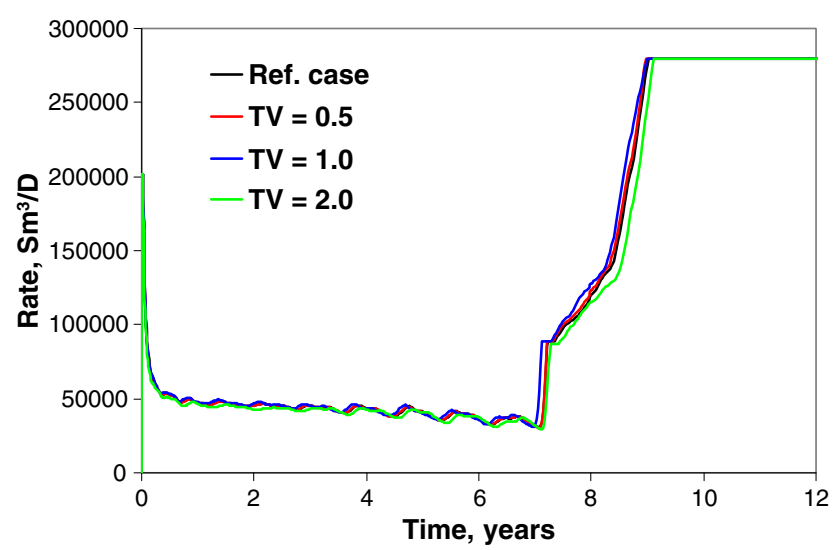

Fig. 27 Field gas rates for three cases with TV $=0.5,1.0$, and 2.0 compared to reference case

\section{Run series 3: optimizing the hor-grid}

Once the decision to use a horizontal grid has been taken, the next question to be addressed is how to define the horizontal layering in an optimal manner. To this extent a number of test cases were run, all using the " $2 \times$ diameter" grid, and a summary of the results will be presented in this section. Close to 100 cases were run, and as it is not practical to discuss these in detail in this paper, the results will be presented in form of a statistical analysis. This is done out of convenience, and the conclusions which are stated below are all based on comparison of the individual runs. However, this comparison matched the "ranking" obtained with the statistical analysis very well.

One of the runs was chosen as a reference case (one resembling the BASEHOR case). In the following we will denote a case as "good" or "bad" based on how closely it resembles the reference case, although this is highly imprecise.

To enable presentation of the conclusions in a concise form, a total variation variable $T V$ will be used. The results to base the comparisons on were chosen as well rates for oil, water, and gas for all wells: the result vector $R V$ is the set \{oil rate, water rate, and gas rate from wells HOPN, HOPE, HOPSE, and HOPW\}.
Then, using $R_{V}$ to denote (normalized) results from the reference case, and $r_{V}$ for corresponding results from the comparison case,

$$
T V=\frac{1}{N} \sum_{V \in R V} \sum_{t=1}^{N}\left[r_{V}(t)-R_{V}(t)\right]^{2}
$$

where $t$ denotes the time variable, taken at 15-day intervals, and $N$ is the number of time steps. The total variation is hence the square difference between the comparison and reference cases, summed for all report steps and all relevant result vectors.

The $T V$ is "just a number", but by comparisons it was found that cases with $T V$ less than unity are "as good as equal", while noticeable differences begin to appear for $T V \approx 2$. An example is shown in Fig. 27.

Note that results and discussion in this section are directly related to the study on the Troll segment, and are mostly not generalizable. To reach similar conclusions for other reservoirs the same kind of sensitivity studies must be carried through for each case.

Oil zone In the reservoir segment in question, the oil zone is $13 \mathrm{~m}$ thick, and the initial choice of using 13 equi-thick layers in the oil zone has worked well. Irrespective of the chosen strategy the layers should be defined such that true perforation depths are honored, i.e. at cell centers. Even a deviation of $0.5 \mathrm{~m}$ from this principle resulted in a difference in simulated gas break-through of one year. Thinner layers than $1 \mathrm{~m}$ did not change the solution noticeably, while $2 \mathrm{~m}$ layers above the perforation layer gave results which were noticeable but not significantly different. The reduction in computing time was however minimal, so very little was gained by increasing layer thickness in the oil zone.

Gas zone All runs in this series were done with the same layering below the gas oil contact; hence any differences are due to the gas zone layering strategy alone. This choice of strategy did not seem to matter at all, as both the results and CPU-times were relatively equal in all the studied cases, even using only two layers in the gas cap. A summary of some of the runs is shown in Table 3.
Table 3 TV and CPU for some gas zone layering alternatives

\begin{tabular}{lclll}
\hline \multicolumn{2}{l}{ Strategy (\# means “number of”) } & & TV & CPU, s \\
\cline { 1 - 2 } \# geo-layers & \multicolumn{2}{l}{ \# hor-layers $(\Delta \mathrm{Z}, \mathrm{GOC}$ upwards) } & & \\
\hline 8 & 10 & $(1.5-2-2.5-7 \times 3)$ & 0.43 & 602 \\
7 & 7 & $(1.5-2.25-3.25-4.5-3 \times 6)$ & 0.39 & 573 \\
0 & 6 & $(1.6-2.6-4.1-6.6-10.5-17)$ & 0.40 & 569 \\
0 & 4 & $(2.5-5-7.5-12.5-15)$ & 0.44 & 534 \\
0 & 2 & $(8-38.5)$ & 0.52 & 513 \\
\hline
\end{tabular}


Table 4 TV and CPU for some water zone layering alternatives (all layers horizontal)

\begin{tabular}{lllc}
\hline \# hor-layers $(\Delta \mathrm{Z}, \mathrm{OWC}$ downwards $)$ & \multicolumn{2}{c}{$\mathrm{TV}$} & $\mathrm{CPU}, \mathrm{s}$ \\
\cline { 1 - 2 }$(m=$ geometric increase factor $)$ & & \\
\hline 15 & $(5 \times 1+10$ layers w. $m=1.5)$ & 1.29 & 243 \\
16 & $(m=1.25$ for all layers $)$ & 1.21 & 235 \\
21 & $(5 \times 1+16$ layers w. $m=1.25)$ & 1.02 & 291 \\
24 & $(1-1.25-1.5-1.75-6 \times 2-2.5-3.1-3.9-4.9-6-7.5 \ldots)$ & 1.15 & 273 \\
26 & $(10 \times 1+16$ layers w. $m=1.25)$ & 0.93 & 372 \\
32 & $(5 \times 1-10 \times 1.5-2.25-3-3.9-4.9-6 \ldots)$ & 1.00 & 361 \\
\hline
\end{tabular}

This somewhat surprising result can be explained by the gas cap being in expansion mode, such that very little if any of the GOC movement is upwards into the gas zone. The initial gas cap is therefore effectively a one-phase domain. As high resolution primarily is needed in fluid contact areas (two- and three-phase domains), it is sufficient to model the gas zone with coarse cells in this study. This conclusion should not be generalized as it will certainly not be valid in situations where the initial GOC does move upwards.

Water zone From experience it is known that the initial OWC on Troll moves both upwards and downwards during production. Hence we should expect that a fine grid is needed in a depth range above and below the initial OWC to capture the contact movement. This was confirmed by the water zone sensitivity batch of runs. All runs in this series were done with identical layering strategy in the gas and oil zones: eight horizontal layers in the gas zone and 13 in the oil zone.

Table 4 summarizes some of the results from cases with only horizontal layers in the water zone (no geolayers).

The total variation is larger than in the gas sensitivity study-none of these cases are particularly good or bad. As expected the best results were achieved when a higher resolution was used near the initial OWC.

As the results from all of the cases with all water zone layers horizontal were only acceptable, but not especially good, the question is whether it is a better strategy to use a hybrid grid in the water zone. (All

Table $5 \mathrm{TV}$ and CPU for strategies varying total thickness of water zone horizontal layers

\begin{tabular}{lllll}
\hline $\begin{array}{l}\text { Hor-grid } \\
\text { thickness }\end{array}$ & \# hor-layers & \# geo-layers & TV & CPU, s \\
\hline 5 & 5 & 56 & 1.57 & 497 \\
16.5 & 9 & 51 & 0.78 & 435 \\
24.5 & 10 & 46 & 0.45 & 402 \\
32.5 & 11 & 40 & 0.48 & 367 \\
47.5 & 12 & 31 & 0.63 & 327 \\
72 & 13 & 12 & 1.02 & 274 \\
102.5 & 14 & 5 & 1.25 & 257 \\
\hline
\end{tabular}

these cases had relatively thick layers in the deepest parts of the reservoir).

The next batch of runs was sensitivities on distribution of horizontal versus geo-layers in the water zone. In these runs;

- Oil and gas zones were modeled as in the previous batch of runs

- From OWC downwards, $\Delta Z=1 \mathrm{~m}$ in the top five layers, then $\Delta Z$ increases by $m=1.5$ down to the base of the horizontal section

- The deepest part of the water zone was modeled with geo-layers

Table 5 summarizes some of the results, which are also shown graphically in Fig. 28.

From these runs, there appears to be an "optimal" thickness of the horizontal grid in the water zone. At least it is clear that better results were obtained when using a combination of horizontal and geo-layers, than with horizontal layers only. Intuitively we would perhaps expect that the deepest part of the water zone should act as an "energy tank", where a detailed description was not necessary. However, the runs clearly show that also the deepest parts of the water zone have impact on the flow pattern, probably due to the need for resolving the pressure distribution. The actual dividing line is not critical; from the figure above it would appear that a water zone horizontal grid thick-

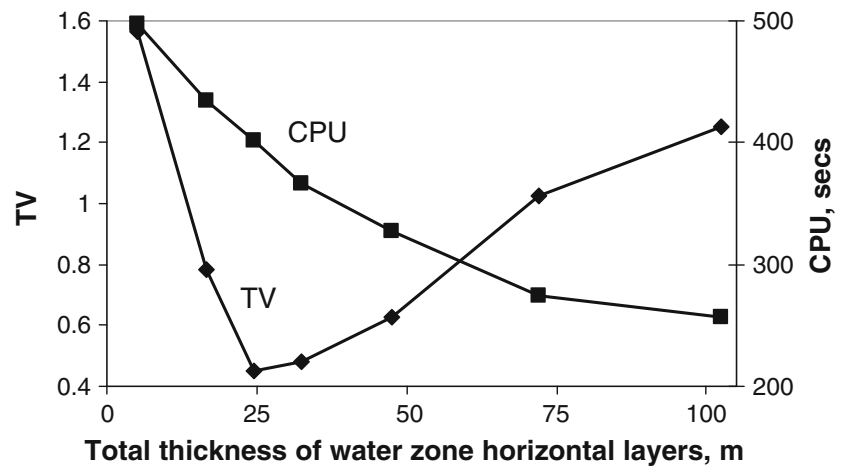

Fig. 28 TV and CPU-time for cases with varying distribution of horizontal vs. geo-layers in the water zone 
Table 6 TV and CPU for some other sensitivities

\begin{tabular}{lll}
\hline Strategy & TV & CPU, s \\
\hline $\begin{array}{l}\text { Hor. grid only in oil zone, } \\
\text { geo-grid for the rest }\end{array}$ & 2.0 & 588 \\
$\begin{array}{l}\text { Geo-grid: top gas cap }-4 \text { m below GOC } \\
\quad+\text { entire water zone. } 9 \text { hor. layers in rest of oil zone. }\end{array}$ & 2.1 & 610 \\
$\begin{array}{l}\text { As case above, except water } \\
\text { zone was optimal hybrid }\end{array}$ & 2.4 & 563 \\
$\begin{array}{l}\text { Hor. grid only for horizontal well layer, } \\
\text { geo-grid for the rest }\end{array}$ & 2.3 & 562 \\
$\begin{array}{l}\text { 6 equi-thick hor. layers in oil zone } \\
\quad \Rightarrow \text { wells } 0.6 \text { m shallower than true depth) }\end{array}$ & 9.5 & 137 \\
\hline
\end{tabular}

was more than an order of magnitude larger than for the horizontal grid, and also suffered from numerical instabilities.

The open question is whether the findings for the models presented in this paper are valid in more general settings. The crucial factor is whether the conversion of petrophysics from geo-based to horizontal grid can be done such that the flow characteristics are preserved. The most challenging case is when the reservoir contains many thin, sloping layers with alternating high and low permeability. For very thin layers with significant dip very small and thin horizontal cells would be needed to resolve the variation, if at all possible. In the Troll study, the permeability variation was of the alternating kind, with moderately thick layers and a moderate dipping angle. The results which were obtained is a strong indication that the horizontal grid was actually able to resolve the petrophysics with required accuracy. For more homogeneous reservoirs with smoother permeability variation or smaller contrasts the conversion to horizontal grid should be at least as reliable as in the Troll segment case. Hence, the horizontal grid concept should be applicable to many thin oil zone reservoirs.

\section{Epilogue}

By the first quarter of 2011, the Troll organization had built a new full-field oil-zone simulation model using a hybrid grid. This model, comprising 1.6 million cells, is the first simulation model in the field's history with acceptable grid resolution over the entire oil zone. In agreement with pre-build requirements, the model can be run with less than one day computing time. As per April $201180 \%$ of the wells have been history matched [16].

\section{Nomenclature}

GOC gas-oil contact

OWC oil-water contact 


\begin{tabular}{|c|c|}
\hline LGR & local grid refinement \\
\hline$D X, D Y, D Z$ & Cell diameter in $x, y, z$-direction \\
\hline$T X, T Y, T Z$ & Transmissibilities ( $x, y, z$-direction) \\
\hline MULTX / Y / Z & $\begin{array}{l}\text { transmissibility multiplier in } x, y \text {, } \\
z \text {-direction }\end{array}$ \\
\hline NNC & non-neighbor connection \\
\hline TV & total variation \\
\hline geo-grid & grid with layers aligned w/geology \\
\hline hor-grid & grid with true horizontal layers \\
\hline ybrid grid & grid with both geo- and hor. layers \\
\hline$n x, n y, n z$ & $\begin{array}{l}\text { number of cells in the grid, in } x, y \text {, } \\
z \text {-direction }\end{array}$ \\
\hline$k_{n}$ & layer $\mathrm{n}$ in hor-grid \\
\hline$K_{n}$ & layer $\mathrm{n}$ in geo-grid \\
\hline & geometric increase factor \\
\hline$K X^{\mathrm{Geo}}$ & Permeability along geo-grid $x$-axis \\
\hline$K X^{\text {Hor }}$ & Permeability along hor-grid $x$-axis \\
\hline
\end{tabular}

Acknowledgements The author would like to thank the Troll partnership (ConocoPhillips Skandinavia AS, Petoro AS, A/S Norske Shell, Statoil Petroleum AS, and Total E\&P Norge AS) for permission to publish this work. The results and opinions presented in this paper do not necessarily reflect the view of the Troll partnership. Funding and support for the project from Statoil/Troll is highly appreciated.

Open Access This article is distributed under the terms of the Creative Commons Attribution Noncommercial License which permits any noncommercial use, distribution, and reproduction in any medium, provided the original author(s) and source are credited.

\section{References}

1. Bagheri, M., Settari, A.: Methods for modeling full tensor permeability in reservoir simulators. J. Can. Pet. Technol. 46(3), 31-38 (2007)

2. Bowen, G.R., Leiknes, J.: Parallel processing applied to local grid refinement. In: Paper SPE 30203 Presented at Petroleum Computer Conference, Houston, 11-14 June 1995
3. Durlofsky, L.J.: Numerical calculation of equivalent grid block permeability tensors for heterogeneous porous media. Water Resour. Res. 27(5), 699-708 (1991)

4. Fantoft, S., Krogh, P.K., Pollen, S.: Evaluation of oil recovery from a thin oil column by a horizontal well in the Oseberg gamma north reservoir. In: Paper SPE 18341 Presented at SPE European Petroleum Conference, London, 16-19 Oct 1988

5. Henriquez, A., Apeland, O.J., Lie, Ø., Cheshire, I.: Novel simulation techniques used in a gas reservoir with a thin oil zone: Troll field. SPE Reserv. Eng. 7(4), 414-418 (1992)

6. Hsu, H.-H.: Use of new horizontal grids in reservoir simulation models improve the chance of success in developing marginal thin oil rim reservoirs using horizontal wells. In: Paper SPE 39548 Presented at SPE India Oil and Gas Conference and Exhibition, New Delhi, 7-9 April 1998

7. Kabir, C. S., Agamini, M., Holguin, R.A.: Production strategy for thin-oil columns in saturated reservoirs. SPE Reserv. Evalu. Eng. 11(1), 73-82 (2008)

8. Kasap, E., Lake, L.W.: Calculating the effective permeability tensor of a gridblock. SPE Form. Eval. 5(2), 192-200 (1990)

9. Lien, S.C., Seines, K., Havig, S.O., Kydland, T.: The first longterm horizontal-well test in the Troll thin oil zone. J. Pet. Technol. 43(8), 914-917, 970-973 (1991)

10. Mlacnik, J.L., Durlofsky, L.J., Heinemann, Z.E.: Sequentially adapted flow-based PEBI grids for reservoir simulation. SPE J. 11, 304-316 (2006)

11. Mundal, S.S., Keilegavlen, E., Aavatsmark, I.: Simulation of anisotropic heterogeneous near-well flow using MPFA methods on flexible grids. Comput. Geosci. 14, 509-525 (2010)

12. Schlumberger: ECLIPSE reference manual 2010. Houston, Schlumberger (2010)

13. Seines, K., Lien, S.C., Haug, B.T.: Troll horizontal well tests demonstrate large production potential from thin oil zones. SPE Reserv. Eval. Eng. 9(2), 133-130 (1994)

14. Thakur, S.C., Bally, K., Therry, D., Simon, L.: Performance of horizontal wells in a thin oil zone between a gas cap and an aquifer, immortelle field, trinidad. In: Paper SPE 36752 Presented at SPE Annual Technical Conference and Exhibition, Denver, 6-9 Oct 1996

15. Toronoyi, R.M., Farouq Ali, S.M.: Determining interblock transmissibility in reservoir simulators. J. Pet. Technol. 26(1), 77-78 (1974)

16. Vinje, J., Nyb $\varnothing$, R., Grinestaff, G.: A new simulation grid type demonstrated for the giant Troll field. In: Presentation at SPE-Bergen One-Day Seminar, Bergen, Norway, 6 April 2011 